\title{
Outer automorphism groups of some ergodic equivalence relations
}

\author{
Alex Furman*
}

\begin{abstract}
Let $\mathrm{R}$ a be countable ergodic equivalence relation of type $\mathrm{II}_{1}$ on a standard probability space $(X, \mu)$. The group Out $\mathrm{R}$ of outer automorphisms of $\mathrm{R}$ consists of all invertible Borel measure preserving maps of the space which map R-classes to R-classes modulo those which preserve almost every $R$-class. We analyze the group Out $R$ for relations $R$ generated by actions of higher rank lattices, providing general conditions on finiteness and triviality of Out $R$ and explicitly computing Out $\mathrm{R}$ for the standard actions. The method is based on Zimmer's superrigidity for measurable cocycles, Ratner's theorem and Gromov's Measure Equivalence construction.
\end{abstract}

Mathematics Subject Classification (2000). 37A20, 28D15, 22E40, 22F50, 46L40.

Keywords. Ergodic equivalence relations, higher rank Lie group, lattices, outer automorphisms.

\section{Introduction and statement of the main results}

Let $(X, \mathscr{B})$ be a standard Borel space with a non-atomic probability Lebesgue measure and let $\mathrm{R}$ be a countable measurable relation of type $\mathrm{II}_{1}$ on $(X, \mathcal{B}, \mu)$, i.e. measurable, countable, ergodic and measure preserving equivalence relation $\mathrm{R} \subset X \times X$. For the abstract definition of this notion the reader is referred to the fundamental work of Feldman and Moore [1], which in particular demonstrates that any such equivalence relation can be presented as the orbit relation

$$
\mathrm{R}_{X, \Gamma}=\{(x, y) \in X \times X \mid \Gamma \cdot x=\Gamma \cdot y\}
$$

of an ergodic, measure preserving action of some countable group $\Gamma$ on the space $(X, \mathcal{B}, \mu)$. In most of the examples in this paper equivalence relations are defined by ergodic measure-preserving actions of concrete countable groups $\Gamma$, namely irreducible lattices in semi-simple connected higher rank real Lie groups.

\footnotetext{
*Supported in part by NSF grants DMS-0049069 and DMS-0094245.
} 
In the purely measure-theoretical context of this paper all objects are considered modulo sets of zero $\mu$-measure, denoted $(\bmod 0)$. Following this convention the measure space automorphism group $\operatorname{Aut}(X, \mu)$ is the group of all invertible Borel maps $T: X \rightarrow X$ with $T_{*} \mu=\mu$, where two such maps which agree on a set of full $\mu$-measure are identified. In a similar fashion two equivalence relations $\mathrm{R}, \mathrm{R}^{\prime}$ on $(X, \mu)$ are identified if there exists a subset $X^{\prime} \subseteq X$ with $\mu\left(X^{\prime}\right)=1$ on which the restrictions of $R$ and $R^{\prime}$ coincide.

Given an equivalence relation $\mathrm{R}$ on $(X, \mu)$ consider the group of relation automorphisms

$$
\text { Aut } \mathrm{R}=\{T \in \operatorname{Aut}(X, \mu) \mid T \times T(\mathrm{R})=\mathrm{R}\}
$$

and the subgroup Inn $\mathrm{R}$ of inner automorphisms, also known as the full group of $\mathrm{R}$, consisting of such $T \in \operatorname{Aut}(X, \mu)$ that $(x, T x) \in \mathrm{R}$ for $\mu$-a.e. $x \in X$. The full group Inn $R$ is normal in Aut $R$ and the outer automorphism group Out $\mathrm{R}$ is defined as the quotient

$$
1 \longrightarrow \text { Inn R } \longrightarrow \text { Aut } \mathrm{R} \stackrel{\epsilon}{\longrightarrow} \text { Out } \mathrm{R} \longrightarrow 1 .
$$

Elements of Out $\mathrm{R}$ represent measurable ways to permute $\mathrm{R}$-classes on $(X, \mu)$. The full group Inn $R$ is always very large (see Lemma 2.1). For the unique amenable equivalence relation $R_{a m}$ of type $I_{1}$ the outer automorphism group Out $R_{a m}$ is also enormous. The purpose of this paper is to analyze Out $\mathrm{R}_{X, \Gamma}$ for orbit relations $\mathrm{R}_{X, \Gamma}$ generated by m.p. ergodic actions of higher rank lattices, in particular presenting many natural examples of relations $R$ with trivial Out $R$. Such examples were first constructed by S. Gefter in [6], [7] (Theorem 1.5 below).

Prior to stating the results let us define two special subgroups in Out $R$, in the case where $\mathrm{R}$ is the orbit relation $\mathrm{R}_{X, \Gamma}$ generated by some measure-preserving action $(X, \mu, \Gamma)$ of some countable group $\Gamma$. In such a situation consider the group $\operatorname{Aut}(X, \Gamma)$ of action automorphisms of the system $(X, \mu, \Gamma)$

$$
\operatorname{Aut}(X, \Gamma):=\{T \in \operatorname{Aut}(X, \mu) \mid T(\gamma \cdot x)=\gamma \cdot T(x) \text { for all } \gamma \in \Gamma\} .
$$

This is the centralizer of $\Gamma$ in $\operatorname{Aut}(X, \mu)$. For a group automorphism $\tau \in$ Aut $\Gamma$ define

$$
\operatorname{Aut}^{\tau}(X, \Gamma):=\left\{T \in \operatorname{Aut}(X, \mu) \mid T(\gamma \cdot x)=\gamma^{\tau} \cdot T(x)\right\}
$$

and let $\operatorname{Aut}^{*}(X, \Gamma)$ be the union of $\operatorname{Aut}^{\tau}(X, \Gamma)$ over $\tau \in$ Aut $\Gamma$. (If the $\Gamma$-action is faithful $\operatorname{Aut}^{*}(X, \Gamma)$ is the normalizer of $\Gamma$ in $\left.\operatorname{Aut}(X, \mu)\right)$. We shall denote by $\mathrm{A}(X, \Gamma)$ and $\mathrm{A}^{*}(X, \Gamma)$ the images of the groups $\operatorname{Aut}(X, \Gamma)$ and $\operatorname{Aut}^{*}(X, \Gamma)$ under the factor map Aut $\mathrm{R}_{X, \Gamma} \stackrel{\epsilon}{\longrightarrow}$ Out $\mathrm{R}_{X, \Gamma}$. Observe that the $\epsilon$-image in Out $\mathrm{R}_{X, \Gamma}$ of the coset $\operatorname{Aut}^{\tau}(X, \Gamma)$ depends only on the outer class $[\tau] \in$ Out $\Gamma$ and therefore can be denoted by $\mathrm{A}^{[\tau]}(X, \Gamma)$. The group $\mathrm{A}(X, \Gamma)$ is normal in $\mathrm{A}^{*}(X, \Gamma)$ and the factor group $\mathrm{A}^{*}(X, \Gamma) / \mathrm{A}(X, \Gamma)$ is (a factor of) a subgroup of Out $\Gamma$. In general, the subgroups 
$\mathrm{A}(X, \Gamma) \subseteq \mathrm{A}^{*}(X, \Gamma)$ of Out $\mathrm{R}_{X, \Gamma}$ depend on the specific presentation of the relation $\mathrm{R}$ as the orbit relation $\mathrm{R}_{X, \Gamma}$ of an action $(X, \mu, \Gamma)$.

In this paper we are mostly interested in ergodic m.p. actions of higher rank lattices and will be using the following terminology and notations:

- For locally compact, secondly countable group $G$ a left-invariant Haar measure will be denoted by $m_{G}$. If $\Gamma \subset G$ is a discrete group so that $G / \Gamma$ carries a finite $G$-invariant measure we say that $\Gamma$ forms a lattice in $G$ and will denote by $m_{G / \Gamma}$ the unique $G$-invariant probability measure on $G / \Gamma$.

- The term semi-simple Lie group will mean semi-simple, connected, center-free, real Lie group $G=\prod G_{i}$ without non-trivial compact factors, unless stated otherwise. A lattice $\Gamma$ in a semi-simple Lie group $G=\prod G_{i}$ is irreducible if $\Gamma$ does not contain a finite index subgroup $\Gamma^{\prime}$ which splits as a direct product of lattices in subfactors. By higher rank lattice hereafter we shall mean an irreducible lattice in a semi-simple Lie group $G$ with $\operatorname{rk}_{\mathbb{R}}(G) \geq 2$.

- A measure-preserving action $(X, \mu, \Gamma)$ of a lattice $\Gamma$ in a semi-simple Lie group $G=\prod G_{i}$ is irreducible if the action of every simple factor $G_{i}$ in the induced $G$-action on $\left(G \times_{\Gamma} X, m_{G / \Gamma} \times \mu\right)$ is ergodic. Clearly, if $G$ is simple then any lattice $\Gamma \subset G$ is irreducible and any ergodic $\Gamma$-action is irreducible.

- For an arbitrary group $\Gamma$ a m.p. action $\left(X_{0}, \mu_{0}, \Gamma\right)$ is a ( $\Gamma$-equivariant) quotient of another m.p. action $(X, \mu, \Gamma)$ if there exists a measurable map $\pi: X \rightarrow X_{0}$ with $\pi_{*} \mu=\mu_{0}$ and $\pi(\gamma \cdot x)=\gamma \cdot \pi(x)$ for $\mu$-a.e. $x \in X$ and all $\gamma \in \Gamma$.

- A measure-preserving action $(X, \mu, \Gamma)$ of an arbitrary group $\Gamma$ is called aperiodic if every finite atomic quotient of $(X, \mu, \Gamma)$ is trivial; equivalently if every finite index subgroup $\Gamma^{\prime} \subset \Gamma$ acts ergodically on $(X, \mu)$.

Remarks. (a) Every mixing ergodic action $(X, \mu, \Gamma)$ of an irreducible lattice $\Gamma$ in a semi-simple Lie group $G$ is irreducible and aperiodic.

(b) By the result of Stuck and Zimmer [14] any ergodic non-atomic m.p. action of an irreducible lattice $\Gamma$ in a semi-simple Lie group $G$ with property (T) is free (mod 0$)$. Recall that a higher rank semi-simple $G$ has property (T) iff it does not have simple factors locally isomorphic to $\mathrm{SO}(n, 1)$ or $\mathrm{SU}(n, 1)$.

(c) For any free, ergodic action $(X, \mu, \Gamma)$ of an irreducible lattice $\Gamma$ in a semisimple Lie group $G$ the map

$$
\operatorname{Aut}(X, \Gamma) \stackrel{\epsilon}{\longrightarrow} \mathrm{A}(X, \Gamma)
$$

is an isomorphism and the homomorphism

$$
\mathrm{A}^{*}(X, \Gamma) / \mathrm{A}(X, \Gamma) \longrightarrow \text { Out } \Gamma
$$

is an embedding (Lemma 2.3 below). 
(d) It follows from the Strong Rigidity (Mostow, Prasad, Margulis) that for an irreducible lattice $\Gamma \subset G \not \mathrm{SL}_{2}(\mathbb{R})$ the automorphism group Aut $\Gamma$ is isomorphic to the normalizer $N_{\text {Aut } G}(\Gamma)$ of $\Gamma$ in Aut $G \supseteq \operatorname{Ad} G \cong G$. Since $\Gamma_{*}:=N_{\text {Aut } G}(\Gamma) \supseteq \Gamma$ is a closed subgroup properly contained in Aut $G$, it forms a lattice in Aut $G$, and Out $\Gamma=\Gamma_{*} / \Gamma$ is always finite.

Thus for an irreducible aperiodic free m.p. action of a higher rank lattice $\Gamma$ the analysis of Out $\mathrm{R}_{X, \Gamma}$ reduces to the analysis of the quotient Out $\mathrm{R}_{X, \Gamma} / \mathrm{A}^{*}(X, \Gamma)$ and the subgroup $\mathrm{A}^{*}(X, \Gamma)$ which, up to at most finite index, is isomorphic to $\operatorname{Aut}(X, \Gamma)$.

Theorem 1.1. Let $G$ be a semi-simple, connected, center-free, real Lie group without non-trivial compact factors and with $\operatorname{rk}_{\mathbb{R}}(G) \geq 2$. Let $\Gamma \subset G$ be an irreducible lattice and $(X, \mu, \Gamma)$ be a measure preserving, ergodic, irreducible, aperiodic, essentially free $\Gamma$-action. Assume that $(X, \mu, \Gamma)$ does not admit measurable $\Gamma$-equivariant quotients of the form $\left(G / \Gamma^{\prime}, m_{G / \Gamma^{\prime}}, \Gamma\right)$ where $\Gamma^{\prime} \subset G$ is a lattice isomorphic to $\Gamma$ and $\Gamma$ acts by $\gamma: g \Gamma^{\prime} \mapsto \gamma g \Gamma^{\prime}$. Then

$$
\text { Out } \mathrm{R}_{X, \Gamma}=\mathrm{A}^{*}(X, \Gamma)
$$

while $\mathrm{A}^{*}(X, \Gamma) \cong \operatorname{Aut}(X, \Gamma) / \Gamma$.

More generally, we have:

Theorem 1.2. Let $\Gamma \subset G$ be a higher rank lattice as in Theorem 1.1 and $(X, \mu, \Gamma)$ be any measure preserving, ergodic, irreducible, aperiodic, essentially free $\Gamma$-action. If $\mathrm{A}^{*}(X, \Gamma)$ has finite index $n>1$ in Out $\mathrm{R}_{X, \Gamma}$ then $(X, \mu, \Gamma)$ has an equivariant measurable quotient

$$
\pi:(X, \mu) \longrightarrow\left(G^{n-1} / \Gamma^{n-1}, m_{G^{n-1} / \Gamma^{n-1}}\right)=\prod_{i=1}^{n-1}\left(G / \Gamma, m_{G / \Gamma}\right)
$$

where the $\Gamma$-action on $\left(G^{n-1} / \Gamma^{n-1}, m_{G^{n-1} / \Gamma^{n-1}}\right)$ is given by

$$
\gamma:\left(x_{i}\right)_{i=1}^{n-1} \mapsto\left(\gamma^{\tau_{i}} \cdot x_{i}\right)_{i=1}^{n-1}
$$

for some fixed automorphisms $\tau_{i} \in$ Aut $G, 1 \leq i<n$.

If $\mathrm{A}^{*}(X, \Gamma)$ has infinite index in Out $\mathrm{R}_{X, \Gamma}$ then $(X, \mu, \Gamma)$ has an infinite product equivariant quotient space

$$
\pi:(X, \mu) \longrightarrow \prod_{i=1}^{\infty}\left(G / \Gamma, m_{G / \Gamma}\right)
$$

with a diagonal $\Gamma$-action on $\gamma:\left(x_{i}\right)_{i=1}^{\infty} \mapsto\left(\gamma^{\tau_{i}} x_{i}\right)_{i=1}^{\infty}$ for some fixed sequence $\tau_{i} \in$ Aut $G, i=1,2, \ldots$. 
Of course, Theorem 1.1 is just a particular case of 1.2 (contrapositive formulation for $n=1)$ since a $\tau$-twisted $\Gamma$-action $\gamma: g \Gamma \mapsto \gamma^{\tau} g \Gamma$ on $\left(G / \Gamma, m_{G / \Gamma}\right)$ is measurably isomorphic to the untwisted $\Gamma$-action $\gamma: g \Gamma^{\prime} \mapsto \gamma g \Gamma^{\prime}$ where $\Gamma^{\prime}=\tau^{-1}(\Gamma)$.

For an $d \times d$ matrix $A$ let $\chi(A):=\sum_{i} \log ^{+}\left|\lambda_{i}(A)\right|$, where $\log ^{+} x=\max \{0, \log x\}$ and $\lambda_{i}(A)$ denote the eigenvalues of $A$. Given a semi-simple group $G$ and $d \in \mathbb{N}$ consider all linear representations $\rho: G \rightarrow \mathrm{GL}_{d}(\mathbb{C})$ (there are finitely many equivalence classes for any $d$ ) and let

$$
W_{G}(d):=\max _{\operatorname{dim} \rho=d} \inf _{g \in G} \frac{\chi(\rho(g))}{\chi(\operatorname{Ad}(g))} .
$$

Corollary 1.3. Let $\Gamma \subset G$ and $(X, \mu, \Gamma)$ be as in Theorem 1.2. Denote by $h(X, \gamma)$ the Kolmogorov-Sinai entropy of the single measure-preserving transformation $\gamma$ of $(X, \mu)$. Then

$$
\left[\text { Out } \mathrm{R}_{X, \Gamma}: \mathrm{A}^{*}(X, \Gamma)\right] \leq 1+\inf _{\gamma \in \Gamma} \frac{h(X, \gamma)}{\chi(\operatorname{Ad}(\gamma))}
$$

If $X$ is a compact manifold with a $C^{1}$-action of a higher rank lattice $\Gamma \subset G$ which preserves a probability measure $\mu$ on $X$ so that $(X, \mu, \Gamma)$ is a free $(\bmod 0)$ action which is ergodic, irreducible and aperiodic, then

$$
\text { [Out } \left.\mathrm{R}_{X, \Gamma}: \mathrm{A}^{*}(X, \Gamma)\right] \leq 1+W_{G}(\operatorname{dim}(X)) .
$$

The function $W_{G}$ satisfies $W_{G}(d) \leq d^{2} / 8$.

Remark. Theorem 1.9 below shows that the inequality (1.1) is sharp. However the estimate (1.2) is probably not optimal, with a more plausible one being $1+$ $\operatorname{dim}(X) / \operatorname{dim} \operatorname{Lie}(G)$.

Remark. As we shall see below, groups Out $\mathrm{R}_{X, \Gamma}$ and $\mathrm{A}(X, \Gamma)$ can be very large when considered as abstract groups, but in all cases below the quotient Out $R_{X, \Gamma} / A^{*}(X, \Gamma)$ is either finite or countable. This might be a general property of actions of higher rank lattices. In fact, this property is known for essentially free ergodic actions $(X, \Gamma)$ of groups $\Gamma$ with Kazhdan's property $(\mathrm{T})$. For such groups (and in a slightly more general situation) Gefter and Golodets introduced a natural topology on Out $\mathrm{R}_{X, \Gamma}$ with respect to which Out $\mathrm{R}_{X, \Gamma}$ is a Polish (i.e. complete separable) group and $\mathrm{A}(X, \Gamma)$ is an open subgroup (see [8], Theorem 2.3, and references throughout Section 2).

In specific cases, in particular in the standard examples of algebraic lattice actions, it is possible to compute the groups Out $\mathrm{R}_{X, \Gamma}$ explicitly as we shall do in Theorems 1.4-1.9 below. In Theorems $1.4-1.8$ the systems $(X, \Gamma)$ do not have $G / \Gamma^{\prime}$ as measurable quotients and therefore by Theorem 1.1 we have Out $\mathrm{R}_{X, \Gamma}=\mathrm{A}^{*}(X, \Gamma)=$ $\operatorname{Aut}^{*}(X . \Gamma) / \Gamma$. The latter groups are of algebraic nature, but their explicit descriptions are cumbersome. Thus for readers convenience we have also presented the groups $\operatorname{Aut}(X, \Gamma)$, which have a more transparent appearance and have at most finite $(\leq \mid$ Out $\Gamma \mid)$ index in $A^{*}(X, \Gamma)$. 
Theorem 1.4. Let $G$ be a simple, connected real Lie group with finite center and $\operatorname{rk}_{\mathbb{R}}(G) \geq 2$ and $\rho: G \hookrightarrow \mathrm{SL}_{N}(\mathbb{R})$ be an embedding such that $\rho(G)$ does not have non-trivial fixed vectors and assume that $G$ has a lattice $\Gamma \subset G$ such that $\rho(\Gamma) \subseteq \mathrm{SL}_{N}(\mathbb{Z})$. Then the natural $\Gamma$-action on the torus $\mathbb{T}^{N}=\mathbb{R}^{N} / \mathbb{Z}^{N}$ is ergodic, aperiodic and the orbit relation $\mathrm{R}_{\mathbb{T}^{N}, \Gamma}$ satisfies

$$
\begin{aligned}
& \text { Out } \mathrm{R}_{\mathbb{T}^{N}, \Gamma}=\mathrm{A}^{*}\left(\mathbb{T}^{N}, \Gamma\right) \cong N_{\mathrm{GL}_{N}(\mathbb{Z})}(\rho(\Gamma)) / \rho(\Gamma) \\
& \mathrm{A}\left(\mathbb{T}^{N}, \Gamma\right) \cong \operatorname{Aut}\left(\mathbb{T}^{N}, \Gamma\right) \cong C_{\mathrm{GL}_{N}(\mathbb{R})}(\rho(G)) \cap \mathrm{GL}_{N}(\mathbb{Z}) .
\end{aligned}
$$

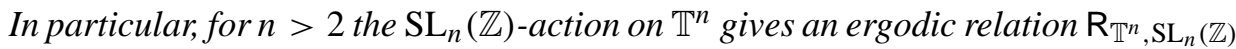
which has no outer automorphisms if $n$ is even, and a single outer automorphism given by $x \mapsto-x$ if $n$ is odd.

Note that in the above theorem we allowed finite non-trivial centers to accommodate the standard example of $\Gamma=\mathrm{SL}_{n}(\mathbb{Z})$ acting on the torus $\mathbb{T}^{n}$ for even $n>2$.

To state the following results we recall the notion of affine transformations of a homogeneous space (these are needed only for the precise description of Out $\mathrm{R}_{X, \Gamma}$ ), however the spirit of the results is captured by the finite index subgroup $\mathrm{A}(X, \Gamma)$ which does not require this notion.

Definition. Let $\Lambda$ be a subgroup of a group $H$, and let $N:=\bigcap_{h \in H} h^{-1} \Lambda h$ denote the maximal subgroup of $\Lambda$ which is normal in $H$. Given an automorphism $\sigma$ of $H / N$ with $\sigma(\Lambda / N)=\Lambda / N$ and $t \in H / N$ denote by $a_{\sigma, t}$ the map

$$
a_{\sigma, t}: h \Lambda \mapsto t \sigma(h) \Lambda
$$

of $H / \Lambda$. Such maps will be called affine, and we shall denote by $\operatorname{Aff}(H / \Lambda)$ the group of all affine maps of $H / \Lambda$.

Replacing $H$ by $H / N$ and $\Lambda$ by $\Lambda / N$ one does not change the homogeneous space: $H / \Lambda \cong(H / N) /(\Lambda / N)$. Thus hereafter we shall assume that $N$ is trivial. Under this assumption the map $(\sigma, t) \mapsto a_{\sigma, t}$ defines an epimorphism $N_{\text {Aut } H}(\Lambda) \ltimes H \longrightarrow \operatorname{Aff}(H / \Lambda)$ (which contains $\{(\operatorname{Ad} \lambda, \lambda) \mid \lambda \in \Lambda\}$ in its kernel) and which maps $H \cong\{\operatorname{Id}\} \times H$ isomorphically onto its image in $\operatorname{Aff}(H / \Lambda)$. This copy of $H$ in $\operatorname{Aff}(H / \Lambda)$ has index bounded by $\mid$ Out $H \mid$.

We shall be interested in situations where some group (a higher rank lattice) $\Gamma$ is embedded in $H, \rho: \Gamma \rightarrow H$, and acts on the homogeneous space $H / \Lambda$ by left translations. Then the normalizer $N_{\operatorname{Aff}(H / \Lambda)}(\rho(\Gamma))$ in $\operatorname{Aff}(H / \Lambda)$ of this action consists of those affine maps $a_{\sigma, t}$ for which $\sigma \in$ Aut $H$ and $t \in H$ satisfy

$$
\sigma(\Lambda)=\Lambda \quad \text { and } \quad \sigma(\rho(\Gamma))=t^{-1} \rho(\Gamma) t
$$

In any case this group contains $N_{H}(\rho(\Gamma))$ as a subgroup of an index bounded by |Out $H \mid$. 
Theorem 1.5 (cf. Gefter [7]). Let $\Gamma$ be a higher rank lattice which admits a dense embedding $\rho: \Gamma \rightarrow K$ into a compact connected Lie group $K$. Then for every closed subgroup $\{e\} \subseteq L \subset K$ the $\Gamma$-action on $\left(K / L, m_{K / L}\right)$ is ergodic, irreducible and aperiodic and the orbit relation $\mathrm{R}_{K / L, \Gamma}$ satisfies

$$
\begin{aligned}
\text { Out } \mathrm{R}_{(K / L, \Gamma)} & =\mathrm{A}^{*}(K / L, \Gamma) \cong N_{\mathrm{Aff}(K / L)}(\rho(\Gamma)) / \rho(\Gamma) \\
\mathrm{A}(K / L, \Gamma) & \cong \operatorname{Aut}(K / L, \Gamma) \cong N_{K}(L) / L .
\end{aligned}
$$

In particular, if the compact group $K$ has no outer automorphisms which normalize $L$ or if $\Gamma$ has no outer automorphisms, then

$$
\text { Out } \mathrm{R}_{K / L, \Gamma} \cong N_{K}(L) / L \text {. }
$$

Remark. A variant of Theorem 1.5 was proved by S. Gefter in [7]. This gave the first example of type $\mathrm{II}_{1}$ equivalence relations without outer automorphisms (see also Corollary 1.8, Theorem 1.10 and the remarks that follow below). Indeed, by a well known arithmetic construction (cf. [17], 5.2.12) certain lattices $\Gamma \subset G:=\operatorname{SO}(p, q)$ admit dense embeddings into the compact group $K:=\mathrm{SO}(n)$ where $n=p+q$. Take $p>q \geq 2$ to ensure $\operatorname{rk}_{\mathbb{R}}(G) \geq 2$ and let $L \cong \mathrm{SO}(n-1)$ be the stabilizer of a point in $K=\mathrm{SO}(n)$ action on the sphere $S^{n-1}$. Then $N_{K}(L)=L$ and since $\mathrm{SO}(n)$ has no outer automorphisms, $\operatorname{Aff}(K / L) \cong N_{K}(L)$, which shows that Out $\mathrm{R}_{(K / L, \Gamma)}$ is trivial.

In Theorem 1.5 the compact group $K$ is taken to be connected to guarantee aperiodicity of the action. Higher rank lattices can also be densely embedded in other compact groups, namely profinite ones. Such embeddings give rise to ergodic actions which strongly violate aperiodicity condition - they are inverse limits of finite quotients. A typical example is the standard embedding

$$
\Gamma:=\operatorname{PSL}_{n}(\mathbb{Z}) \stackrel{\rho}{\longrightarrow} K:=\operatorname{PSL}_{n}\left(\mathbb{Z}_{p}\right) .
$$

It was observed in [7] (Remark 2.8) that in this case Out $\mathrm{R}_{K, \Gamma}$ contains a group isomorphic to $\operatorname{PSL}_{n}\left(\mathbb{Q}_{p}\right)$, in such a way that

$$
\mathrm{A}(K, \Gamma) \cong K=\mathrm{PSL}_{n}\left(\mathbb{Z}_{p}\right) \subset \mathrm{PSL}_{n}\left(\mathbb{Q}_{p}\right) \subseteq \text { Out } \mathrm{R}_{K, \Gamma}
$$

so that $\mathrm{A}(K, \Gamma)$ has infinite index in Out $\mathrm{R}_{K, \Gamma}$. We claim that the last inclusion is essentially an equality. More generally, the following result holds:

Theorem 1.6. Consider the natural dense embedding of $\Gamma=\operatorname{PSL}_{n}(\mathbb{Z}), n \geq 3$, in the profinite group $K=\prod_{i=1}^{r} \operatorname{PSL}_{n}\left(\mathbb{Z}_{p_{i}}\right)$ where $\left\{p_{1}, \ldots, p_{r}\right\}$ is a finite set of distinct primes. Then Out $\mathrm{R}_{K, \Gamma}$ is a $\mathbb{Z} / 2$ extension of

$$
H=\prod_{i=1}^{r} \operatorname{PSL}_{n}\left(\mathbb{Q}_{p_{i}}\right)
$$


with the transpose map $\left(k_{1}, \ldots, k_{r}\right) \mapsto\left(k_{1}^{t}, \ldots, k_{r}^{t}\right)$ of $K$ giving rise to the $\mathbb{Z} / 2$ extension.

Another family of standard examples is described by the following

Theorem 1.7. Let $\Gamma \subset G$ be a higher rank lattice as in Theorem 1.1, $H$ be a connected Lie group, $\Lambda \subset H$ be a closed subgroup so that $H / \Lambda$ carries an $H$-invariant probability measure $m_{H / \Lambda}$, and assume that $H$ does not admit surjective homomorphisms $\sigma: H \rightarrow G$ with $\sigma(\Lambda) \subseteq \Gamma$. Suppose that there exists a homomorphism $\rho: G \rightarrow H$ such that each of the simple factors $G_{i}$ of $G$ acts ergodically on $\left(H / \Lambda, m_{H / \Lambda}\right)$. Then for the $\Gamma$-action on $\left(H / \Lambda, m_{H / \Lambda}\right)$ one has

$$
\begin{aligned}
\text { Out } \mathrm{R}_{(H / \Lambda, \Gamma)} & =\mathrm{A}^{*}(H / \Lambda, \Gamma) \cong N_{\mathrm{Aff}(H / \Lambda)}(\rho(\Gamma)) / \rho(\Gamma) \\
\mathrm{A}(H / \Lambda, \Gamma) & \cong \operatorname{Aut}(H / \Lambda, \Gamma) \cong C_{\mathrm{Aff}(H / \Lambda)}(\rho(\Gamma)) .
\end{aligned}
$$

Corollary 1.8. Let $\Gamma \subset G$ be a higher rank lattice as in Theorem 1.1, $H$ be a connected semi-simple Lie group with trivial center, $\rho: G \hookrightarrow H$ be an embedding and let $\Lambda \subset H$ be an irreducible lattice. Assume that either $\rho$ is a proper embedding, i.e. $G \not H$, or that $\rho: G \rightarrow H$ is an isomorphism but $\Lambda$ is not abstractly isomorphic to a subgroup of finite index in $\Gamma$. Then the $\Gamma$-action on $\left(H / \Lambda, m_{H / \Lambda}\right)$ by left translations is ergodic, irreducible and aperiodic (in fact mixing) and the orbit relation $\mathrm{R}_{H / \Lambda, \Gamma}$ has

$$
\text { Out } \mathrm{R}_{(H / \Lambda, \Gamma)}=\mathrm{A}^{*}(H / \Lambda, \Gamma) \cong N_{\mathrm{Aff}(H / \Lambda)}(\rho(\Gamma)) / \rho(\Gamma) .
$$

This group contains the centralizer $C_{H}(\rho(G))$ as a normal subgroup of finite index dividing $\mid$ Out $\Lambda|\cdot|$ Out $\Gamma \mid$.

Remark. Corollary 1.8 also allows to construct ergodic equivalence relations without outer automorphisms. Indeed if a simple Lie group $G \not \mathrm{SL}_{2}(\mathbb{R})$ has no outer automorphisms, then maximal lattices $\Gamma$ in $G$ have trivial Out $\Gamma$ as well. Choosing two non-commensurable maximal lattices $\Gamma, \Lambda$ in such a $G$ one obtains an equivalence relation $\mathrm{R}_{G / \Lambda, \Gamma}$ without outer automorphisms. Similarly, one can find proper embeddings $G \subset H$ where $G$ and $H$ are simple higher rank Lie groups with Out $G$, Out $H, C_{H}(G)$ all being trivial. Then for any choice of maximal lattices $\Gamma \subset G$, $\Lambda \subset H$, the $\Gamma$-action on $H / \Lambda$ gives $\mathrm{R}_{H / \Lambda, \Gamma}$ without outer automorphisms.

All the examples discussed so far had the property that the original system $(X, \mu, \Gamma)$ did not admit measurable $\Gamma$-equivariant quotients of the form $\left(G / \Gamma^{\prime}, m_{G / \Gamma^{\prime}}, \Gamma\right)$; and therefore Theorem 1.1 allowed to conclude that

$$
\text { Out } \mathrm{R}_{X, \Gamma}=\mathrm{A}^{*}(X, \Gamma) \cong \operatorname{Aut}^{*}(X, \Gamma) / \Gamma .
$$

The following result analyzes what happens if this assumption is not satisfied. 
Theorem 1.9. Let $\Gamma \subset G$ be a higher rank lattice as in Theorem 1.1 and let $\Gamma$-act on $\left(G / \Gamma, m_{G / \Gamma}\right)$ by left translations. Then for the corresponding orbit relation $\mathrm{R}_{G / \Gamma, \Gamma}$

$$
\begin{aligned}
& \text { [Out } \mathrm{R}_{G / \Gamma, \Gamma}\left.: \mathrm{A}^{*}(G / \Gamma, \Gamma)\right]=2 \\
& \mathrm{~A}(G / \Gamma, \Gamma) \cong \operatorname{Aut}(G / \Gamma, \Gamma) \cong\{1\} \\
& \mathrm{A}^{*}(G / \Gamma, \Gamma) \cong \text { Out } \Gamma \\
& \text { Out } \mathrm{R}_{(G / \Gamma, \Gamma)} \cong(\mathbb{Z} / 2 \mathbb{Z}) \times \text { Out } \Gamma .
\end{aligned}
$$

More generally, for any $n \in \mathbb{N}$ the diagonal left $\Gamma$-action on the product space $\left(G^{n} / \Gamma^{n}, m_{G^{n}} / \Gamma^{n}\right)$ satisfies

$$
\begin{aligned}
& \text { [Out } \mathrm{R}_{\left(G^{n} / \Gamma^{n}, \Gamma\right)}\left.: \mathrm{A}^{*}\left(G^{n} / \Gamma^{n}, \Gamma\right)\right]=n+1 \\
& \mathrm{~A}\left(G^{n} / \Gamma^{n}, \Gamma\right) \cong \operatorname{Aut}\left(G^{n} / \Gamma^{n}, \Gamma\right) \cong S_{n} \\
& \mathrm{~A}^{*}\left(G^{n} / \Gamma^{n}, \Gamma\right) \cong S_{n} \times \text { Out } \Gamma \\
& \text { Out } \mathrm{R}_{\left(G^{n} / \Gamma^{n}, \Gamma\right)} \cong S_{n+1} \times \text { Out } \Gamma
\end{aligned}
$$

where $S_{n}$ denotes the permutation group on $\{1, \ldots, n\}$.

For the diagonal $\Gamma$-action on the infinite product $(X, \mu)=\left(G / \Gamma, m_{G / \Gamma}\right)^{\mathbb{Z}}$, the index $\left[\right.$ Out $\mathrm{R}_{(X, \Gamma)}: \mathrm{A}^{*}(X, \Gamma)$ ] is infinite countable

$$
\begin{aligned}
& \mathrm{A}(X, \Gamma) \cong \operatorname{Aut}(X, \Gamma) \cong S_{\infty} \\
& \mathrm{A}^{*}(X, \Gamma) \cong S_{\infty} \times \text { Out } \Gamma \\
& \text { Out } \mathrm{R}_{(X, \Gamma)} \cong S_{\infty+1} \times \text { Out } \Gamma
\end{aligned}
$$

where $S_{\infty}$ denotes the full permutation group on $\mathbb{Z}$, and $S_{\infty+1}$ the permutation group of $\mathbb{Z} \cup\{p t\}$ to suggest that the embedding $\mathrm{A}^{*}(X, \Gamma) \subset$ Out $\mathrm{R}_{X, \Gamma}$ corresponds to the natural embedding $S_{\infty} \subset S_{\infty+1}$ direct product with Out $\Gamma$.

Let $\mathrm{R}$ be an ergodic $\mathrm{II}_{1}$-relation on a probability space $(X, \mu)$, and $E \subset X$ be a measurable subset with $\mu(E)>0$. The restriction $\mathrm{R}_{E}:=\mathrm{R} \cap(E \times E)$ of $\mathrm{R}$ to $E$ is a II 1 -ergodic relation with respect to the normalized measure $\mu_{E}:=\left.\mu(E)^{-1} \cdot \mu\right|_{E}$. Since Inn R acts transitively on subsets of the same size (Lemma 2.1) for any $F \subset X$ with $\mu(F)=\mu(E)$ the relation $\mathrm{R}_{F}$ on $\left(F, \mu_{F}\right)$ is isomorphic to $\mathrm{R}_{E}$ on $\left(E, \mu_{E}\right)$. Hence given a $\mathrm{II}_{1}$-relation $\mathrm{R}$, for every $0<t \leq 1$ there is a well defined, up to isomorphism, ergodic $\mathrm{II}_{1}$-relation $\mathrm{R}_{t}$ obtained from $\mathrm{R}$ by restriction to a subset of measure $t$. (Realizing $(X, \mu)$ as the unit interval $[0,1]$ one may think of $\mathrm{R}_{t}$ as the restriction of $\mathrm{R}$ to the sub-interval $[0, t])$.

If $\mathrm{R}$ has an additional property that $\mathrm{R}_{t} ¥ \mathrm{R}_{s}$ for all $0<t \neq s \leq 1$, one says that $\mathrm{R}$ has a trivial fundamental group. Orbit relations $\mathrm{R}=\mathrm{R}_{X, \Gamma}$ generated by free, ergodic, irreducible m.p. actions of higher rank lattices $\Gamma$ always have trivial fundamental groups (cf. Gefter and Golodec [8]). Recent work [5] of Gaboriau gives other classes of such relations. 
Regardless whether the fundamental group of $R$ is trivial or not, all restricted relations $\mathrm{R}_{t}$ obtained from a given ergodic $\mathrm{II}_{1}$-relation $\mathrm{R}$ have the same outer automorphism group: Out $\mathrm{R}_{t} \cong$ Out $\mathrm{R}$ (see Lemma 2.2). Hence

Theorem 1.10. Let $\Gamma \subset G$ and $(X, \mu, \Gamma)$ be as in Theorem 1.2. For $0<t \leq 1$ let $\mathrm{R}_{t}$ denote the (isomorphism class of) equivalence relation obtained from $\mathrm{R}:=\mathrm{R}_{X, \Gamma}$ by a restriction to a subset $E_{t} \subset X$ of measure $\mu\left(E_{t}\right)=t$. Then $\left\{\mathrm{R}_{t}\right\}_{0<t \leq 1}$ is a family of mutually non-isomorphic ergodic equivalence relations of type $\mathrm{II}_{1}$ with the same outer automorphism group Out $\mathrm{R}_{t} \cong$ Out $\mathrm{R}_{X, \Gamma}$. In particular, there exist uncountablymany mutually non-isomorphic ergodic relations with trivial outer automorphism groups.

Remarks. (a) In [3], Theorems D(1)-(2), it is shown that for an ergodic action $(X, \mu, \Gamma)$ of a lattice $\Gamma$ in a simple higher rank Lie group $G$, there is a countable set $M_{X, \Gamma} \subset \mathbb{R}$ so that for $t \in(0,1) \backslash M_{X, \Gamma}$ the relation $\mathrm{R}_{t}$ cannot be generated by a free $(\bmod 0)$ action of any group. Therefore Theorem 1.10 provides a variety of examples of such exotic relations without outer automorphisms.

(b) In a recent work [11] Monod and Shalom develop a new type of "higher rank" superrigidity theorems for products of hyperbolic-like groups. Using this new tool and the methods of the current paper Monod and Shalom construct uncountably many non weakly equivalent relations $\mathrm{R}$ of type $\mathrm{II}_{1}$ with trivial Out $\mathrm{R}$ (see [11], Theorem 1.12).

Organization of the paper. Section 2 contains some general facts about $\mathrm{II}_{1}$-relations. In Section 3 we discuss the Measure Equivalence point of view which provides a convenient framework for the study of Out $\mathrm{R}_{X, \Gamma} / \mathrm{A}^{*}(X, \Gamma)$. Special features of higher rank lattices, especially superrigidity for cocycles, are used in Section 4 in a construction of $\Gamma$-equivariant standard quotients $\pi:(X, \mu) \rightarrow\left(G / \Gamma, m_{G / \Gamma}\right)$ associated to every $[T] \in$ Out $\mathrm{R}_{X, \Gamma} \backslash \mathrm{A}^{*}(X, \Gamma)$, which provide the proof of Theorem 1.1. In Section 5 we recall some ergodic-theoretic applications of Ratner's theorem for actions on homogeneous spaces. These results are used in Section 6 to assemble the standard quotients for the proof of Theorem 1.2, and in Sections 7 and 8 to compute the outer automorphism groups for the standard examples. Section 9 contains the proof of Theorem 1.6.

\section{Generalities}

Let $\mathrm{R}$ be an ergodic $\mathrm{II}_{1}$ relation on a non-atomic probability space $(X, \mu)$. For readers convenience we include the proof of the following standard fact

Lemma 2.1. For every measurable $E, F \subseteq X$ with $\mu(E)=\mu(F)>0$ there exists $T \in \operatorname{Inn} \mathrm{R}$ so that $\mu(T E \triangle F)=0$. 
Proof. By [1], Theorem 1, there exists an action $(X, \mu, \Gamma)$ of some countable group $\Gamma$ so that $\mathrm{R}=\mathrm{R}_{X, \Gamma}$. Such an action is necessarily measure-preserving and ergodic. For any measurable subsets $A, B \subseteq X$ let $c(A, B):=\sup _{\gamma} \mu(\gamma A \cap B)$. Ergodicity implies that $c(A, B)>0$ whenever $\mu(A)>0$ and $\mu(B)>0$. Let $E_{0}:=E, F_{0}:=F$ and define by induction on $n \geq 1$ measurable sets $E_{n}, F_{n} \subseteq X$ and elements $\gamma_{n} \in \Gamma$ as follows: given $E_{n}, F_{n}$ choose $\gamma_{n}$ so that

$$
\mu\left(\gamma_{n} E_{n} \cap F_{n}\right) \geq c\left(E_{n}, F_{n}\right) / 2
$$

and let $E_{n+1}:=E_{n} \backslash \gamma_{n}^{-1} F_{n}, F_{n+1}:=F_{n} \backslash \gamma_{n} E_{n}$. Set $E_{\infty}:=\cap E_{n}, F_{\infty}:=\cap F_{n}$. We have $\mu\left(E_{\infty}\right)=\mu\left(F_{\infty}\right)$ because $\mu\left(E_{n}\right)=\mu\left(F_{n}\right)$ for all finite $n$. In fact $\mu\left(E_{\infty}\right)=$ $\mu\left(F_{\infty}\right)=0$. Indeed, otherwise one would have $c\left(E_{n}, F_{n}\right) \geq c:=c\left(E_{\infty}, F_{\infty}\right)>0$ for all $n$, contrary to the choice of $\gamma_{n}$ at the stage where $\mu\left(E_{n} \backslash E_{n+1}\right)<c / 2$. Hence $E_{n}^{\prime}:=E_{n} \backslash E_{n+1}$ and $F_{n}^{\prime}:=F_{n} \backslash F_{n+1}$ constitute measurable partitions of $E$ and $F$ respectively. Defining $T(x)$ to be $\gamma_{n} \cdot x$ if $x \in E_{n}^{\prime}$ and $T(x)=x$ for $x \notin E$, we get the desired $T \in \operatorname{Inn} \mathrm{R}$.

Given an ergodic $\mathrm{II}_{1}$-relation $\mathrm{R}$ on $(X, \mu)$, and a positive measure subset $E \subseteq X$ we denote by $\mathrm{R}_{E}$ the restricted relation $\mathrm{R} \cap(E \times E)$ on $\left(E, \mu_{E}\right)$, where $\mu_{E}=$ $\left.\mu(E)^{-1} \cdot \mu\right|_{E}$.

Lemma 2.2. For a measurable set $E \subseteq X$ with $\mu(E)>0$

$$
\text { Out } \mathrm{R}_{E} \cong \text { Out } \mathrm{R} \text {. }
$$

Proof. First observe that any $T \in$ Aut $\mathrm{R}_{E}$ can be extended to a $\bar{T} \in$ Aut $\mathrm{R}$. To see this choose some measurable partition $X=E \cup X_{1} \cup \cdots \cup X_{N}$ so that $0<\mu\left(X_{i}\right) \leq \mu(E)$; and choose measurable subsets $E_{i} \subseteq E$ with $\mu\left(E_{i}\right)=\mu\left(X_{i}\right)$. By Lemma 2.1 there exist $S_{i}, R_{i} \in \operatorname{Inn} \mathrm{R}$ so that $S_{i}\left(X_{i}\right)=E_{i}$ and $R_{i}\left(X_{i}\right)=T\left(E_{i}\right)$. Define $\bar{T}$ by $\bar{T}(x)=R_{i}^{-1} \circ T \circ S_{i}(x)$ for $x \in X_{i}$ and $\bar{T}(x)=T(x)$ for $x \in E$ to get a desired $\bar{T} \in$ Aut R.

This extension procedure is well defined on the level of outer classes. In other words if $\bar{T}, \bar{S} \in$ Aut R are some extensions of some $T, S \in$ Aut $_{E}$, then $[T]=[S] \in$ Out $\mathrm{R}_{E}$ iff $[\bar{T}]=[\bar{S}] \in$ Out R. Indeed for $\mu$-a.e. $x \in X$ choose $y \in E$ so that $x \sim y$ and observe that

$$
\bar{T}(x) \sim \bar{T}(y)=T(y) \quad \text { and } \quad S(y)=\bar{S}(y) \sim \bar{S}(x) .
$$

Hence $\bar{T}(x) \sim \bar{S}(x)$ for $\mu$-a.e. $x \in X$ iff $T(y) \sim S(y)$ for $\mu_{E}$-a.e. $y \in E$.

Thus there is a well defined injective map Out $\mathrm{R}_{E} \rightarrow$ Out $\mathrm{R}$, which is easily seen to be a homomorphism of groups. To verify its surjectivity, note that given any $\bar{T} \in$ Aut $\mathrm{R}$ there is an $\bar{S} \in \operatorname{Inn} \mathrm{R}$ with $\bar{S}(\bar{T}(E))=E$. Thus $\bar{T}^{\prime}:=\bar{S} \circ \bar{T}$ maps $E$ to itself, and $[\bar{T}]=\left[\bar{T}^{\prime}\right] \in$ Out $\mathrm{R}$ appears as an extension of $\left[\left.T^{\prime}\right|_{E}\right] \in$ Out $\mathrm{R}_{E}$. 
For the rest of the section we consider a free $(\bmod 0)$ ergodic m.p. action $(X, \mu, \Gamma)$ of some countable group $\Gamma$, denoting by $\mathrm{R}_{X, \Gamma}$ the corresponding orbit relation.

Lemma 2.3 (Gefter [7], Lemmas 2.6, 3.2). Let $(X, \mu, \Gamma)$ be a free m.p. ergodic action of a countable group $\Gamma$.

(a) If $\Gamma$ has Infinite Conjugacy Classes then $\operatorname{Aut}(X, \Gamma) \stackrel{\epsilon}{\longrightarrow} \mathrm{A}(X, \Gamma)$ is an isomorphism.

(b) If $\Gamma$ has the property that any $\tau \in$ Aut $\Gamma$ with $\gamma^{\tau}=\gamma$ on a finite index subgroup $\gamma \in \Gamma_{0} \subseteq \Gamma$ has to be the identity, then

$$
\operatorname{Ker}\left(\operatorname{Aut}^{*}(X, \Gamma) \stackrel{\epsilon}{\longrightarrow} \mathrm{A}^{*}(X, \Gamma)\right)=\{x \mapsto \gamma \cdot x\}_{\gamma \in \Gamma} \cong \Gamma .
$$

In particular, the conclusions of (a) and (b) hold for any free ergodic action $(X, \mu, \Gamma)$ of an irreducible lattice $\Gamma$ in a semi-simple Lie group $G \not \mathrm{SL}_{2}(\mathbb{R})$.

Proof. (a) Any $T \in \operatorname{Aut}(X, \Gamma) \cap \operatorname{Inn} \mathrm{R}_{X, \Gamma}$ has the form $T: x \rightarrow \xi_{x} \cdot x$ for some measurable $x \mapsto \xi_{x} \in \Gamma$ and satisfies $T(\gamma \cdot x)=\gamma \cdot T(x)$. Hence

$$
\gamma \xi_{x} \cdot x=\gamma \cdot T(x)=T(\gamma \cdot x)=\xi_{\gamma \cdot x} \gamma \cdot x
$$

which gives $\xi_{\gamma \cdot x}=\gamma \xi_{x} \gamma^{-1}$ because the action is assumed to be free $(\bmod 0)$. Thus the distribution $\xi_{*} \mu$ of $\xi_{x}$ on $\Gamma$ is conjugation invariant, and therefore is uniform on finite conjugacy classes of $\Gamma$, i.e. supported on $e$. Hence $T(x)=x$ and $\operatorname{Ker}(\operatorname{Aut}(X, \Gamma) \stackrel{\epsilon}{\longrightarrow} \mathrm{A}(X, \Gamma))$ is trivial.

(b) Any $T \in \operatorname{Aut}^{\tau}(X, \Gamma) \cap \operatorname{Inn} \mathrm{R}_{X, \Gamma}$ satisfies

$$
T(x)=\xi_{x} \cdot x, \quad T(\gamma \cdot x)=\gamma^{\tau} \cdot T(x)
$$

which gives $\xi_{\gamma \cdot x}=\gamma^{\tau} \xi_{x} \gamma^{-1}$. For $\xi \in \Gamma$ let $E_{\xi}:=\left\{x \in X \mid \xi_{x}=\xi\right\}$. Then $\gamma E_{\xi}=E_{\gamma^{\tau} \xi \gamma^{-1}}$. Observe that for $\xi \neq \xi^{\prime} \in \Gamma$ one has $\mu\left(E_{\xi} \cap E_{\xi^{\prime}}\right)=0$ because the action is free $(\bmod 0)$. Hence choosing $\xi_{0} \in \Gamma$ with $\mu\left(E_{\xi_{0}}\right)>0$ we have $\gamma^{\tau} \xi_{0} \gamma^{-1}=\xi_{0}$ (equivalently $\xi_{0}^{-1} \gamma^{\tau} \xi_{0}=\gamma$ ) for all $\gamma$ in a finite index subgroup $\Gamma_{0} \subseteq \Gamma$. It follows from the assumption that $\gamma^{\tau}=\xi_{0} \gamma \xi_{0}^{-1}$ for all $\gamma \in \Gamma$, so that $T: x \mapsto \xi_{0} \cdot x$.

Finally, for an irreducible lattice $\Gamma \subset G \not \mathrm{SL}_{2}(\mathbb{R})$ the ICC is a standard fact (easy for the group $G$ itself and follows for $\Gamma$ using Borel's density theorem), while the condition for (b) follows from the Strong Rigidity Theorem.

Given $T \in$ Aut $\mathrm{R}_{X, \Gamma}$ define a measurable map $\alpha_{T}: \Gamma \times X \rightarrow \Gamma$ by

$$
T(\gamma \cdot x)=\alpha_{T}(\gamma, x) \cdot T(x) .
$$


Note that $\alpha_{T}(\gamma, x)$ is well defined $(\bmod 0)$ due to the freeness assumption on the action. Furthermore, one easily verifies the cocycle property

$$
\alpha_{T}\left(\gamma_{2} \gamma_{1}, x\right)=\alpha_{T}\left(\gamma_{2}, \gamma_{1} \cdot x\right) \alpha_{T}\left(\gamma_{1}, x\right)
$$

for all $\gamma_{1}, \gamma_{2} \in \Gamma$ and $\mu$-a.e. $x \in X$. The cocycle $\alpha_{T}: \Gamma \times X \rightarrow \Gamma$ will be called the rearrangement cocycle associated to $T \in$ Aut $\mathrm{R}_{X, \Gamma}$. Rearrangement cocycles (as opposed to general ones) have the following special property: for $\mu$-a.e. $x \in X$ the correspondence $\gamma \in \Gamma \mapsto \alpha_{T}(\gamma, x) \in \Gamma$ is a permutation of $\Gamma$ elements.

Two (general) cocycles $\alpha, \beta: \Gamma \times X \rightarrow \Gamma$ are said to be cohomologous in $\Gamma$ if there exists a measurable map $x \mapsto \xi_{x} \in \Gamma$, such that

$$
\alpha(\gamma, x)=\xi_{\gamma \cdot x}^{-1} \beta(\gamma, x) \xi_{x}
$$

for all $\gamma \in \Gamma$ and $\mu$-a.e. $x \in X$. We denote by $[\alpha]_{\Gamma}$ the equivalence class of all measurable cocycles cohomologous (in $\Gamma$ ) to $\alpha$. Note the very special cocycle $c_{1}: \Gamma \times X \rightarrow \Gamma$ given by $c_{1}(\gamma, x)=\gamma$, and for a general $\tau \in$ Aut $\Gamma$ let $c_{\tau}: \Gamma \times X \rightarrow$ $\Gamma$ stand for the cocycle $c_{\tau}(\gamma, x)=\gamma^{\tau}$.

Proposition 2.4. Let $T, S$ in Aut $\mathrm{R}_{X, \Gamma}$ be relation automorphisms, [T], $[S]$ in Out $\mathrm{R}_{X, \Gamma}$ the corresponding classes, and let $\alpha_{T}, \alpha_{S}: \Gamma \times X \rightarrow \Gamma$ denote the associated rearrangement cocycles. Then

(a) $\alpha_{T \circ S}(\gamma, x)=\alpha_{T}\left(\alpha_{S}(\gamma, x), S(x)\right)$.

(b) $\alpha_{T}=c_{1} \Leftrightarrow T \in \operatorname{Aut}(X, \Gamma)$.

(c) $\alpha_{T}=c_{\tau} \Leftrightarrow T \in \operatorname{Aut}^{\tau}(X, \Gamma)$.

(d) $\left[\alpha_{T}\right]_{\Gamma}=\left[c_{\tau}\right]_{\Gamma} \Leftrightarrow[T] \in \mathrm{A}^{[\tau]}(X, \Gamma)$.

Proof. For $T, S \in$ Aut $\mathrm{R}_{X, \Gamma}$ compute

$$
T \circ S(\gamma \cdot x)=T\left(\alpha_{S}(\gamma, x) \cdot S(x)\right)=\alpha_{T}\left(\alpha_{S}(\gamma, x), S(x)\right) \cdot T(S(x)) .
$$

This proves (a). Statements (b) and (c) follow from the definitions.

Proof of (d). Any $[T] \in \mathrm{A}^{[\tau]}(X, \Gamma)$ can be represented by $T=A \circ J$ where $A \in \operatorname{Aut}^{\tau}(X, \Gamma)$ and $J \in \operatorname{Inn} \mathrm{R}_{\Gamma}$ is given by $J: x \mapsto \xi_{x}^{-1} \cdot x$. Then for all $\gamma \in \Gamma$ and $\mu$-a.e. $x \in X$

$$
\begin{aligned}
T(\gamma \cdot x) & =A\left(\xi_{\gamma \cdot x}^{-1} \gamma \cdot x\right)=\left(\xi_{\gamma \cdot x}^{-1} \gamma\right)^{\tau} \cdot A(x) \\
& =\left(\xi_{\gamma \cdot x}^{-1}\right)^{\tau} \gamma^{\tau} \xi_{x}^{\tau} \cdot A\left(\xi_{x}^{-1} \cdot x\right)=\zeta_{\gamma \cdot x}^{-1} \gamma^{\tau} \zeta_{x} \cdot T(x)
\end{aligned}
$$

where $\zeta_{x}=\left(\xi_{x}\right)^{\tau} \in \Gamma$. Hence

$$
\alpha_{T}(\gamma, x)=\zeta_{\gamma \cdot x}^{-1} \gamma^{\tau} \zeta_{x}
$$

and $\left[\alpha_{T}\right]_{\Gamma}=\left[c_{\tau}\right]_{\Gamma}$. 
On the other hand, assuming that the rearrangement cocycle $\alpha_{T}$ associated with $T \in$ Aut $\mathrm{R}_{\Gamma}$ satisfies (2.2) for some $\tau \in$ Aut $\Gamma$ and a measurable $x \mapsto \zeta_{x} \in \Gamma$, set $\xi_{x}=\left(\zeta_{x}\right)^{\tau^{-1}}$ and consider the map $A: X \rightarrow X$, defined by $A(x):=\zeta_{x} \cdot T(x)$. We have

$$
\begin{aligned}
A(\gamma \cdot x) & =\zeta_{\gamma \cdot x} \cdot T(\gamma \cdot x)=\zeta_{\gamma \cdot x} \zeta_{\gamma \cdot x}^{-1} \gamma^{\tau} \zeta_{x} \cdot T(x) \\
& =\gamma^{\tau} \cdot\left(\zeta_{x} \cdot T(x)\right)=\gamma^{\tau} \cdot A(x) .
\end{aligned}
$$

The pushforward measure $A_{*} \mu$ is absolutely continuous with respect to $\mu$ (recall that $\Gamma$ is countable) and $\Gamma$-invariant. Ergodicity of the action implies that $A_{*} \mu=\mu$, so that $A$ is invertible. Thus $A \in \operatorname{Aut}^{\tau}(X, \Gamma)$, while the map $J:=A^{-1} \circ T$ is a measure space automorphism. Since

$$
\xi_{x} \cdot J(x)=\xi_{x} \cdot A^{-1}(T(x))=A^{-1}\left(\zeta_{x} \cdot T(x)\right)=x
$$

the map $J(x)=\xi_{x}^{-1} \cdot x$ is an inner automorphism.

\section{Measure Equivalence point of view}

The following notion of Measure Equivalence Coupling, introduced by Gromov in [9], 0.5.E, and considered in [2] and [3] by the author, provides a very convenient point of view on orbit relation automorphisms.

Definition. A Measure Equivalence Coupling of two (infinite) countable groups $\Gamma$ and $\Lambda$ is an (infinite) Lebesgue measure space $(\Omega, m)$ with two commuting, free, measure preserving actions of $\Gamma$ and $\Lambda$, such that each of the actions has a finite measure fundamental domain.

We shall use left and right notations for the $\Gamma$ and $\Lambda$ actions

$$
\gamma: \omega \mapsto \gamma \omega, \quad \lambda: \omega \mapsto \omega \lambda
$$

in order to emphasize that the actions commute. For our current purposes we shall only need self ME-couplings $(\Omega, m)$ of $\Gamma$, i.e. Measure Equivalence Couplings of $\Gamma$ with itself. Given such a coupling $(\Omega, m)$ let $X, Y \subset \Omega$ be some fundamental domains for the right and the left $\Gamma$-actions on $(\Omega, m)$ respectively. Define the associated measurable maps

$$
\lambda=\lambda_{X}: \Gamma \times X \rightarrow \Gamma, \quad \rho=\rho_{Y}: Y \times \Gamma \rightarrow \Gamma
$$

by requiring that for a.e. $x \in X$ (resp. $y \in Y$ ) one has $\gamma x \in X \lambda(\gamma, x)$ (resp. $y \gamma \in \rho(y, \gamma) Y$ ). The left $\Gamma$-action on $\Omega / \Gamma$ (resp. the right $\Gamma$-action on $\Gamma \backslash \Omega$ ), always denoted by a dot ".", can be identified with the measure preserving $\Gamma$-action 
on $X$ with the finite Lebesgue measure $m_{X}=\left.m\right|_{X}$ (resp. on $Y$ with $m_{Y}=\left.m\right|_{X}$ ) defined by

$$
\gamma \cdot x=\gamma x \lambda(\gamma, x)^{-1}, \quad y \cdot \gamma=\rho(y, \gamma)^{-1} y \gamma .
$$

With respect to these left and right $\Gamma$-actions $\lambda_{X}$ and $\rho_{Y}$ become measurable left and right cocycles respectively, namely satisfy:

$$
\lambda\left(\gamma_{1} \gamma_{2}, x\right)=\lambda\left(\gamma_{1}, \gamma_{2} \cdot x\right) \lambda\left(\gamma_{2}, x\right), \quad \rho\left(y, \gamma_{1} \gamma_{2}\right)=\rho\left(y, \gamma_{1}\right) \rho\left(y \cdot \gamma_{1}, \gamma_{2}\right) .
$$

We shall say that a self ME-coupling $(\Omega, m)$ is ergodic if the $\Gamma$-action on $\left(X,\left.m\right|_{X}\right)$ is ergodic, which is equivalent to the ergodicity of the $\Gamma \times \Gamma$-action on the infinite space $(\Omega, m)$ (see [2], Lemma 2.2).

With the fundamental domain $X \subset \Omega$ for $\Omega / \Gamma$ being fixed, all fundamental domains $X^{\prime} \subset \Omega$ for $\Omega / \Gamma$ are in one-to-one correspondence with measurable maps $x \mapsto \xi_{x} \in \Gamma$ : given a fundamental domain $X^{\prime}$ one sets $\xi_{x}=\gamma$, if $x \gamma \in X^{\prime}$, while given a measurable $x \mapsto \xi_{x} \in \Gamma$ one takes

$$
X^{\prime}:=\left\{x \xi_{x} \mid x \in X\right\}
$$

The left $\Gamma$-actions on $X^{\prime}$ and $X$ are naturally identified via $\theta: X \rightarrow X^{\prime}, \theta: x \mapsto x \xi_{x}$, and the cocycles $\lambda_{X}: \Gamma \times X \rightarrow \Gamma, \lambda_{X^{\prime}}: \Gamma \times X^{\prime} \rightarrow \Gamma$ are conjugate

$$
\lambda_{X^{\prime}}(\gamma, \theta(x))=\xi_{\gamma \cdot x}^{-1} \lambda_{X}(\gamma, x) \xi_{x} .
$$

Similar statements hold for the right actions, their fundamental domains and the associated cocycles.

If $X \subset \Omega$ is a fundamental domain for both left and right $\Gamma$-actions, we shall say that $X$ is a two-sided fundamental domain.

Lemma 3.1 (see [3], Theorem 3.3). Let $(\Omega, m)$ be an ergodic self ME-coupling of some group $\Gamma$, and let $X, Y \subset \Omega$ be right and left fundamental domains for $\Omega / \Gamma$ and $\Gamma \backslash \Omega$ respectively. Then $\Omega$ admits a two-sided fundamental domain $Z$ iff $m(X)=m(Y)$.

Proof. Obviously all left fundamental domains have the same $m$-measure and the same holds for right fundamental domains. Thus the existence of a two-sided fundamental domain $Z$ implies $m(X)=m(Z)=m(Y)$. Now assume that $m(X)=m(Y)$. It is well known that ergodic m.p. actions on finite or infinite Lebesgue spaces the full group acts transitively on sets of the same measure (Lemma 2.1 for the finite measure case). Using the ergodicity of the $\Gamma \times \Gamma$-action on $(\Omega, m)$ the condition $m(X)=m(Y)$ implies that there exist measurable partitions $X=\bigcup_{i, j} X_{i, j}, Y=\bigcup_{i, j} Y_{i, j}$, and elements $\gamma_{i}^{\prime} \in \Gamma$ and $\gamma_{j}^{\prime \prime} \in \Gamma$, so that $Y_{i, j}=\gamma_{i}^{\prime-1} X_{i, j} \gamma_{j}^{\prime \prime}$. Then

$$
\bigcup_{i, j} X_{i, j} \gamma_{j}^{\prime \prime} \text { and } \bigcup_{i, j} \gamma_{i}^{\prime} Y_{i, j}
$$


give the same set $Z \subset \Omega$. Being formed by piecewise right $\Gamma$-translates of $X \cong \Omega / \Gamma$, the set $Z$ is a right fundamental domain for $\Omega / \Gamma$; and at the same time being formed by piecewise left $\Gamma$-translates of $Y \cong \Gamma \backslash \Omega$, the same set $Z$ is a left fundamental domain for $\Gamma \backslash \Omega$.

Now consider a free m.p. action $(X, \mu, \Gamma)$ of some countable group $\Gamma$ and let $\mathrm{R}_{X, \Gamma}$ be the corresponding orbit relation. Given $T \in$ Aut $\mathrm{R}_{X, \Gamma}$ consider the infinite measure space $(\Omega, m):=\left(X \times \Gamma, \mu \times m_{\Gamma}\right)$ with two commuting $\Gamma$-actions, as usual written from the left and from the right:

$$
\gamma_{1}(x, \gamma):=\left(\gamma_{1} \cdot x, \alpha_{T}\left(\gamma_{1}, x\right) \gamma\right), \quad(x, \gamma) \gamma_{2}:=\left(x, \gamma \gamma_{2}\right)
$$

where $\alpha_{T}: \Gamma \times X \rightarrow \Gamma$ is the rearrangement cocycle associated with $T \in$ Aut $\mathrm{R}_{X, \Gamma}$. The space $(\Omega, m)$ with thus defined $\Gamma \times \Gamma$-actions forms an ergodic self ME-coupling of $\Gamma$, because $\bar{X}:=X \times\left\{e_{\Gamma}\right\} \subset \Omega$ is a two-sided fundamental domain. The fact that $\bar{X}$ is a right fundamental domain is obvious. To see that $\bar{X}$ is a left fundamental domain recall that for a.e. $x \in X$ the map $\gamma \mapsto \alpha_{T}(\gamma, x)$ is a bijection of $\Gamma$, so for $m$-a.e. $\left(x, \gamma_{1}\right)$ there is a unique $\gamma \in \Gamma$ with $\alpha_{T}(\gamma, x)=\gamma_{1}^{-1}$ which gives

$$
\gamma\left(x, \gamma_{1}\right)=\left(\gamma \cdot x, \alpha_{T}(\gamma, x) \gamma_{1}\right) \in \bar{X}=X \times\{e\} .
$$

Also observe that

$$
\lambda_{\bar{X}}(\gamma, x)=\alpha_{T}(\gamma, x)
$$

Lemma 3.2. Let $(\Omega, m)=\left(X \times \Gamma, \mu \times m_{\Gamma}\right)$ be a selfME-coupling corresponding to $T \in$ Aut $\mathrm{R}_{X, \Gamma}$. There is a one-to-one correspondence between two-sided fundamental domains $\bar{X}^{\prime} \subset \Omega$ and

$$
T^{\prime} \in \text { Aut } \mathrm{R}_{X, \Gamma} \quad \text { with }\left[T^{\prime}\right]=[T] \in \text { Out } \mathrm{R}_{X, \Gamma}
$$

where $\bar{X}^{\prime}=\left\{\left(x, \xi_{x}\right) \mid x \in X\right\}$ corresponds to $T^{\prime}: x \mapsto \xi_{x}^{-1} \cdot T(x)$. Moreover

$$
\alpha_{T^{\prime}}(\gamma, x)=\lambda_{\bar{X}^{\prime}}\left(\gamma,\left(x, \xi_{x}\right)\right)=\xi_{\gamma \cdot x}^{-1} \lambda_{\bar{X}}(\gamma,(x, e)) \xi_{x}=\xi_{\gamma \cdot x}^{-1} \alpha_{T}(\gamma, x) \xi_{x} .
$$

Proof. Suppose that $\bar{X}^{\prime} \subset \Omega=X \times \Gamma$ is a two-sided fundamental domain. The fact that both $\bar{X}=X \times\{e\}$ and $\bar{X}^{\prime}$ are right fundamental domains implies that $\bar{X}^{\prime}$ is of the form $\left\{\left(x, \xi_{x}\right) \mid x \in X\right\}$ for some measurable $\xi: X \rightarrow \Gamma$. In order to verify (3.3) for the map $T^{\prime}: X \rightarrow X, T^{\prime}: x \mapsto \xi_{x}^{-1} \cdot T(x)$, it suffices to check that $T^{\prime}$ is one-to-one $(\bmod 0)$, the relations between the cocycles $\alpha_{T^{\prime}}, \lambda_{\bar{X}^{\prime}}, \lambda_{\bar{X}}$ and $\alpha_{T}$ being straightforward.

Assume that $T^{\prime}(x)=T^{\prime}(y)$ which means $\xi_{x}^{-1} \cdot T(x)=\xi_{y}^{-1} \cdot T(y)$. Then $T(x)$ and $T(y)$ are on the same $\Gamma$-orbit in $X$, and so are $x$ and $y$, i.e. $y=\gamma \cdot x$ for some $\gamma \in \Gamma$. Thus

$$
\xi_{x}^{-1} \cdot T(x)=\xi_{\gamma \cdot x}^{-1} \cdot T(\gamma \cdot x)=\xi_{\gamma \cdot x}^{-1} \alpha_{T}(\gamma, x) \cdot T(x)
$$


which means that $\xi_{\gamma \cdot x}=\alpha_{T}(\gamma, x) \xi_{x}$. In $\Omega$ we have

$$
\gamma\left(x, \xi_{x}\right)=\left(\gamma \cdot x, \alpha_{T}(\gamma, x) \xi_{x}\right)=\left(\gamma \cdot x, \xi_{\gamma \cdot x}\right)
$$

with both $\left(x, \xi_{x}\right)$ and $\left(\gamma \cdot x, \xi_{\gamma \cdot x}\right)$ in $\bar{X}^{\prime}$. Since $\bar{X}^{\prime}$ is a two-sided fundamental domain, in particular a left fundamental domain, it follows that $\gamma=e$ and $x=y$. Hence $T^{\prime}$ is indeed a measure space automorphism of $(X, \mu)$ and the rest of its properties follow automatically. The fact that $T^{\prime}$ as in (3.3) gives rise to a two-sided fundamental domain $\bar{X}^{\prime}$ is proved by back tracking the above argument.

Next consider an equivariant quotient map $\Phi:(\Omega, m) \rightarrow\left(\Omega_{0}, m_{0}\right)$ of self MEcouplings of $\Gamma$, i.e. a measurable map $\Phi: \Omega \rightarrow \Omega_{0}$ such that

$$
\Phi_{*} m=m_{0} \quad \text { and } \quad \Phi\left(\gamma_{1} \omega \gamma_{2}\right)=\gamma_{1} \Phi(\omega) \gamma_{2} .
$$

Observe that the preimage $X:=\Phi^{-1}\left(X_{0}\right)$ (resp. $Y:=\Phi^{-1}\left(Y_{0}\right)$ ) of any right fundamental domain $X_{0} \subset \Omega_{0}$ (resp. any left fundamental domain $Y_{0} \subset \Omega_{0}$ ) is a right (resp. left) fundamental domain in $\Omega$. If $X=\Phi^{-1}\left(X_{0}\right)$ we shall say that $X \subset \Omega$ and $X_{0} \subset \Omega_{0}$ are $\Phi$-compatible. Note also that if $(\Omega, m)$ is an ergodic coupling then so is $\left(\Omega_{0}, m_{0}\right)$, and if $(\Omega, m)$ admits a two-sided fundamental domain then

$$
m_{0}\left(X_{0}\right)=m(X)=m(Y)=m_{0}\left(Y_{0}\right)
$$

so that $\left(\Omega_{0}, m_{0}\right)$ also admits a two-sided fundamental domain $Z_{0}$, and taking $Z:=$ $\Phi^{-1}\left(Z_{0}\right)$ we obtain a two-sided fundamental domain for $(\Omega, m)$ which is $\Phi$-compatible with $Z_{0} \subset \Omega_{0}$.

Observe that for $\Phi$-compatible right fundamental domains $X \subset \Omega$ and $X_{0} \subset \Omega_{0}$ one has

$$
\lambda_{X}(\gamma, \omega)=\lambda_{X_{0}}(\gamma, \Phi(\omega)) .
$$

Realizing the natural left $\Gamma$-action on $(\Omega, m) / \Gamma$ by the $\Gamma$-action

$$
\gamma: x \mapsto \gamma \cdot x=\gamma x \lambda_{X}(\gamma, x)^{-1}
$$

on a $\Phi$-compatible fundamental domain $X \subset \Omega$, one obtains a $\Gamma$-equivariant quotient map $X \stackrel{\Phi}{\longrightarrow} X_{0}$ which is a concrete realization of the left $\Gamma$-equivariant map $(\Omega, m) / \Gamma \rightarrow\left(\Omega_{0}, m_{0}\right) / \Gamma$ defined by $\Phi$. This discussion is summarized by the following

Proposition 3.3. Let $(X, \mu, \Gamma)$ be a free, ergodic, measure preserving action, $T \in$ Aut $\mathrm{R}_{X, \Gamma}$ and let $\left(\Omega_{[T]}, m\right)$ be the corresponding self ME-coupling of $\Gamma$. Assume that $\left(\Omega_{[T]}, m\right)$ has an equivariant quotient ME-coupling $\Phi:\left(\Omega_{[T]}, m\right) \rightarrow\left(\Omega_{0}, m_{0}\right)$. Fix a two sided fundamental domain $X_{0} \subset \Omega_{0}$, denote by $\left(X_{0}, \mu_{0}, \Gamma\right)$ the left $\Gamma$-action on $\left(X_{0}, \mu_{0}\right) \cong\left(\Omega_{0}, m_{0}\right) / \Gamma$, and let

$$
\pi:(X, \mu, \Gamma) \rightarrow\left(X_{0}, \mu_{0}, \Gamma\right)
$$


denote the $\Gamma$-equivariant quotient map induced by $\Phi$. Then there exists a $\hat{T} \in$ Aut $\mathrm{R}_{X, \Gamma}$ with $[T]=[\hat{T}] \in$ Out $\mathrm{R}_{X, \Gamma}$ so that

$$
\alpha_{\hat{T}}(\gamma, x)=\lambda_{X_{0}}(\gamma, \pi(x)) .
$$

\section{Superrigidity and standard quotients}

In this section we specialize to actions of irreducible lattices $\Gamma$ in higher rank semisimple Lie groups $G$.

Proposition 4.1 (see [2], Theorem 4.1). Let G be a semi-simple, connected, centerfree, real Lie group without non-trivial compact factors and with $\mathrm{rk}_{\mathbb{R}}(G) \geq 2$. Let $\Gamma \subset G$ be an irreducible lattice and $(X, \mu, \Gamma)$ be a measure preserving, ergodic, irreducible, essentially free $\Gamma$-action. Given any $T \in$ Aut $\mathrm{R}_{X, \Gamma}$ let $\left(\Omega_{[T]}, m\right)$ be the associated self ME-coupling as in Section 3. Then there exists a well defined class $[\tau] \in$ Out $G$ so that given any representative $\tau$ of $[\tau]$ there exists a measurable map $\Phi: \Omega_{[T]} \rightarrow G$ defined uniquely $(\bmod 0)$ so that

$$
\Phi\left(\gamma_{1} \omega \gamma_{2}\right)=\gamma_{1}^{\tau} \Phi(\omega) \gamma_{2} \quad\left(\gamma_{1}, \gamma_{2} \in \Gamma\right)
$$

and one of the following two alternatives holds:

(a) either $\Phi_{*} m$ coincides with the Haar measure $m_{G}$ on $G$, normalized so that $\Gamma$ has covolume one, or

(b) $\Phi_{*} m$ is an atomic measure of the form

$$
\frac{1}{k} \sum_{i=1}^{k} \sum_{\gamma \in \Gamma} \delta_{g_{i} \gamma}
$$

where $\left\{g_{i}\right\}_{1}^{k} \subset G$ are such that $\left\{g_{1} \Gamma, \ldots, g_{k} \Gamma\right\}$ is a single finite $\tau(\Gamma)$-orbit on $G / \Gamma$. In particular, $\Gamma$ has a subgroup $\Gamma_{1}$ of index $k$ so that $\tau\left(\Gamma_{1}\right)$ has index $k$ in $g_{1} \Gamma g_{1}^{-1}$, and $\tau(\Gamma)$ and $\Gamma$ are commensurable.

If the $\Gamma$-action on $(X, \mu)$ is aperiodic, then either (a) holds or in alternative (b) we have $k=1$ which means that

(b') $\Phi_{*} m$ coincides with the counting measure $m_{\Gamma^{\prime}}$ on $\Gamma^{\prime}=\tau(\Gamma) \subset G$ where $\tau(\Gamma)=g \Gamma g^{-1}$ for some $g \in G$.

This proposition is essentially Theorem 4.1 in [2], the proof of which is based on Zimmer's superrigidity for cocycles and Ratner's theorem. In [2] the statement is formulated in a slightly different form and only for lattices in higher rank simple Lie groups. Since we need some details of the proof to be used later, we include the main arguments here. 
Proof. Fix a $T \in$ Aut $\mathrm{R}_{X, \Gamma}$ representing $[T]$ and consider the rearrangement cocycle $\alpha_{T}: \Gamma \times X \rightarrow \Gamma \subset G$ as a $G$-valued cocycle. This cocycle is Zariski dense in $G$ (this is a form of Borel's density theorem, see [17], p. 99, or [2], Lemma 4.2). Thus the assumption that $\Gamma$ is a higher rank lattice with an irreducible action on $(X, \mu)$ allows to apply Zimmer's superrigidity for measurable cocycles theorem [17] (in [2], Theorem 4.1, we did not use irreducibility of the action and therefore had to restrict the discussion to lattices in higher rank simple groups $G$ ). Hence there exists a Borel map $\phi: X \rightarrow G$ and a homomorphism $\tau: \Gamma \rightarrow G$, so that

$$
\alpha_{T}(\gamma, x)=\phi(\gamma \cdot x)^{-1} \gamma^{\tau} \phi(x)
$$

for $\gamma \in \Gamma$ and $\mu$-a.e. $x \in X$. By Margulis' superrigidity $\tau$ extends to a $G$-automorphism and we denote by $\tau \in$ Aut $G$ this extension. Defining the map

$$
\Phi: \Omega_{[T]}=X \times \Gamma \rightarrow G
$$

by

$$
\Phi(x, \gamma):=\phi(x) \gamma
$$

one verifies

$$
\begin{aligned}
\Phi\left(\gamma_{1}(x, \gamma) \gamma_{2}\right) & =\Phi\left(\gamma_{1} \cdot x, \alpha_{T}\left(\gamma_{1}, x\right) \gamma \gamma_{2}\right)=\phi\left(\gamma_{1} \cdot x\right) \alpha_{T}\left(\gamma_{1}, x\right) \gamma \gamma_{2} \\
& =\phi\left(\gamma_{1} \cdot x\right) \phi\left(\gamma_{1} \cdot x\right)^{-1} \gamma_{1}^{\tau} \phi(x) \gamma \gamma_{2} \\
& =\gamma_{1}^{\tau} \Phi(x, \gamma) \gamma_{2} .
\end{aligned}
$$

Choose $F \subset G$ a Borel fundamental domain for $G / \Gamma$ and let $\bar{X}^{\prime}:=\Phi^{-1}(F)$. Hence $\bar{X}^{\prime} \subset \Omega_{[T]}$ is a fundamental domain for $\Omega_{[T]} / \Gamma$ so that $m\left(\bar{X}^{\prime}\right)=1$. This implies that the pushforward measure $m_{0}:=\Phi_{*} m$ on $G$ has $m_{0}(F)=1$ (in particular $m_{0}$ is finite on compact sets) while the restriction $\left.m_{0}\right|_{F}$ defines a regular Borel probability measure $\mu_{0}$ on $G / \Gamma$, which is invariant and ergodic for the left $\tau(\Gamma)$-action.

An application of Ratner's theorem (see [2], Lemma 4.6, with an easy modification needed to handle semi-simple rather simple Lie groups) implies that $\mu_{0}$ is either (i) $\mu_{0}=m_{G / \Gamma}-$ the normalized Haar measure $m_{G / \Gamma}$, or (ii) is an atomic measure.

In case (i) the map $\Phi$ defined in (4.2) clearly maps $m$ on $\Omega_{[T]}$ to the Haar measure $m_{G}$ as in Proposition 4.1 (a). The uniqueness statements in Proposition 4.1 follow from [2], Theorem 4.1.

In case (ii) the atomic $\tau(\Gamma)$-invariant measure $\mu_{0}$ on $G / \Gamma$ has to be concentrated on a single finite $\tau(\Gamma)$-orbit $\left\{g_{1} \Gamma, \ldots, g_{k} \Gamma\right\}$ with equal weights $1 / k$. Let $\Gamma_{1}$ be the stabilizer of $g_{1} \Gamma \in G / \Gamma$. Then $\left[\Gamma: \Gamma_{1}\right]=k$ and $\tau\left(\Gamma_{1}\right) g_{1} \Gamma=g_{1} \Gamma$ i.e. $\tau\left(\Gamma_{1}\right)$ has index $k$ in $g_{1} \Gamma g_{1}^{-1}$.

The preimage $\Omega_{1}=\Phi^{-1}\left(g_{1} \Gamma\right)$ is $\Gamma_{1} \times \Gamma$-invariant set which gives rise to a measurable $\Gamma_{1}$-invariant subset $X_{1}$ of $X$ with $\mu\left(X_{1}\right)=1 / k$. If $\Gamma$-action on $(X, \mu)$ is aperiodic, then necessarily $k=1$ and $m_{0}=\sum_{\gamma \in \Gamma} \delta_{g \gamma}$ and $\tau(\Gamma)=g \Gamma g^{-1}$. 
Remark. The uniqueness of $\Phi_{[T]}$ in particular implies that the rearrangement cocycle $\alpha_{T}$ can be written in the form (4.1) with the measurable map $\phi: X \rightarrow G$ being uniquely defined $(\bmod 0)$ as soon as a representative $\tau \in$ Aut $G$ of $[\tau] \in$ Out $G$ is chosen. Hereafter this unique "straightening" map $\phi$ will be denoted by $\phi_{T, \tau}$.

Theorem 4.2 (Standard Quotients). Let $G$ be a semi-simple, connected, center-free, real Lie group without non-trivial compact factors and with $\operatorname{rk}_{\mathbb{R}}(G) \geq 2 ; \Gamma \subset G$ an irreducible lattice and $(X, \mu, \Gamma)$ be a measure preserving, ergodic, irreducible, essentially free $\Gamma$-action. Then every $[T] \in$ Out $\mathrm{R}_{X, \Gamma}$ defines a unique class $[\tau] \in$ Out $G$ such that given any representative $\tau \in$ Aut $G$ of $[\tau]$ there is a measurable map $\pi: X \rightarrow G / \Gamma$, defined uniquely $(\bmod 0)$ and satisfying

$$
\pi(\gamma \cdot x)=\tau(\gamma) \cdot \pi(x)
$$

for $\mu$-a.e. $x \in X$ and all $\gamma \in \Gamma$. There are two alternatives.

Either the following equivalent conditions hold:

(a1) the distribution of $\phi_{T, \tau}(x)$ on $G$ is absolutely continuous with respect to the Haar measure $m_{G}$,

(a2) $\pi_{*} \mu=m_{G / \Gamma}$ - the $G$-invariant probability measure on $G / \Gamma$,

(a3) there exists $\hat{T} \in$ Aut $\mathrm{R}_{X, \Gamma}$ with $[\hat{T}]=[T]$ and $\pi(x)=\phi_{\hat{T}, \tau}(x) \Gamma$;

or the following equivalent conditions hold:

(b1) the distribution of $\phi_{T, \tau}(x)$ on $G$ is purely atomic,

(b2) $\pi_{*} \mu=k^{-1} \sum_{1}^{k} \delta_{g_{i}} \Gamma$ where $\left\{g_{1} \Gamma, \ldots, g_{k} \Gamma\right\}$ is a finite $\tau(\Gamma)$-orbit on $G / \Gamma, \Gamma$ contains a subgroup $\Gamma_{1}$ of index $k$ so that $\tau\left(\Gamma_{1}\right)$ is a subgroup of index $k$ in $g_{1} \Gamma g_{1}^{-1}$; and $X_{1}=\pi^{-1}\left(\left\{g_{1} \Gamma\right\}\right)$ is a $\Gamma_{1}$-ergodic components of $(X, \mu)$ with $\mu\left(X_{1}\right)=1 / k$

(b3) there exists $\hat{T} \in$ Aut $\mathrm{R}_{X, \Gamma}$ with $[\hat{T}]=[T]$ and

$$
\phi_{\hat{T}, \tau}(x)=g_{1} \quad \text { for } \mu \text {-a.e. } x \in X_{1} \subset X .
$$

If $\Gamma$-action on $(X, \mu)$ is aperiodic then conditions (a1)-(a3) above are equivalent to (a4) $[T] \notin \mathrm{A}^{*}(X, \Gamma)$,

while their alternatives (b1)-(b3) are equivalent to

(b4) $[T] \in \mathrm{A}^{*}(X, \Gamma)$;

moreover in (b2)-(b3) one has $k=1$ and these conditions take the following form:

(b2') $\pi_{*} \mu=\delta_{g \Gamma}$ where $g \in G$ satisfies $\tau(\Gamma)=g \Gamma g^{-1}$;

(b3') there exists $\hat{T} \in$ Aut $\mathrm{R}_{X, \Gamma}$ with $[\hat{T}]=[T]$ and

$$
\phi_{\hat{T}, \tau}(x)=g \text { for } \mu \text {-a.e. } x \in X \text {. }
$$


Proof. Consider the self ME-coupling $\left(\Omega_{[T]}, m\right)$ with the corresponding outer class $[\tau] \in$ Out $G$. Given a choice $\tau \in$ Aut $G$ of $[\tau]$ let

$$
\Phi: \Omega_{[T]} \rightarrow G
$$

be the $\tau(\Gamma) \times \Gamma$-equivariant map as in Proposition 4.1. Then $\Phi$ uniquely defines a measurable map

$$
\pi:(X, \mu, \Gamma) \cong\left(\Omega_{[T]}, m\right) / \Gamma \rightarrow G / \Gamma, \quad \pi(\gamma \cdot x)=\gamma^{\tau} \cdot \pi(x) .
$$

Let us show that the alternatives (a) and (b) in Proposition 4.1 yield mutually exclusive conditions (a1)-(a3) and (b1)-(b3) respectively.

In case (a) where $\Phi_{*} m=m_{G}$, (a1)-(a3) follow from Proposition 3.3 and the construction (4.2) of $\Phi$.

Case (b): $\Phi_{*} m=k^{-1} \sum_{i=1}^{k} \sum_{\gamma \in \Gamma} \delta_{g_{i} \gamma}$ where $\left\{g_{1} \Gamma, \ldots, g_{k} \Gamma\right\}$ is a single $\tau(\Gamma)$ orbit on $G / \Gamma$. Condition (b1) is clearly satisfied. Let

$$
\Gamma_{i}=\left\{\gamma \in \Gamma \mid \gamma^{\tau} g_{i} \Gamma=g_{i} \Gamma\right\} \quad \text { and } \quad X_{i}=\pi^{-1}\left(\left\{g_{i} \Gamma\right\}\right) .
$$

where $\pi: X \rightarrow\left\{g_{1} \Gamma, \ldots, g_{k} \Gamma\right\}$ is the $\Gamma$-equivariant map above. Then conjugate groups $\Gamma_{i}$ have index $k$ in $\Gamma$, and $\Gamma$ permutes the disjoint sets $X_{i}$ (and so $\mu\left(X_{i}\right)=1 / k$ ) while each $X_{i}$ is $\Gamma_{i}$-invariant for $i=1, \ldots, k$. Moreover $\Gamma_{i}$ acts ergodically on $X_{i}$ because $\mathrm{R}_{X_{i}, \Gamma_{i}}=\mathrm{R}_{X, \Gamma} \cap\left(X_{i} \times X_{i}\right)$. This proves (b2).

The set $X_{0}=\left\{g_{1}, \ldots, g_{k}\right\}$ forms a fundamental domain for the $\tau(\Gamma) \times \Gamma$-action on $G$. The corresponding cocycle $\lambda_{X_{0}}$ satisfies

$$
\lambda_{X_{0}}\left(\gamma_{1}, g_{1}\right)=g_{1}^{-1} \gamma_{1}^{\tau} g_{1} \quad\left(\gamma_{1} \in \Gamma_{1}\right)
$$

Applying Proposition 3.3 we obtain $\hat{T} \in$ Aut $\mathrm{R}_{X, \Gamma}$ with $[\hat{T}]=[T] \in$ Out $\mathrm{R}_{X, \Gamma}$ and

$$
\alpha_{\hat{T}}(\gamma, x)=\lambda_{X_{0}}(\gamma, \pi(x))=g_{1}^{-1} \gamma^{\tau} g_{1}
$$

for all $\gamma \in \Gamma_{1}$ and a.e. $x \in X_{1}=\pi^{-1}\left(\left\{g_{1} \Gamma\right\}\right)=\Phi^{-1}\left(\left\{g_{1}\right\}\right)$. We deduce that $\phi_{\hat{T}, \tau}(x)=g_{1}$ for $x \in X_{1}$, proving (b3).

If the $\Gamma$-action $(X, \mu)$ is aperiodic, one has $k=1$ so that (b2), (b3) take the form of (b2'), (b3'). Condition (b3) follows from (4.3) and Proposition 2.4 (c). The latter also explains why (b4) is incompatible with (a1)-(a3).

Proof of Theorem 1.1. For $\tau \in$ Aut $G$ the $\Gamma$-action on $G / \tau^{-1}(\Gamma)$ is isomorphic to the $\tau$-twisted $\Gamma$-action on $G / \Gamma$, both with the Haar measure. Since $(X, \mu, \Gamma)$ is assumed not to have these actions among its measurable quotients, any $T \in$ Aut $\mathrm{R}_{X, \Gamma}$ fails condition (a2) in Theorem 4.2, while satisfies the alternatives (b1-4), which means that $[T] \in \mathrm{A}^{*}(X, \Gamma)$. 


\section{Some applications of Ratner's Theorem}

In this section we recall some applications of Ratner's Theorem (see [12] and references therein). Note that in these results there are no restrictions on the rank of the semi-simple group $G$. In fact the results remain true whenever $G$ is a connected Lie group generated by Ad-unipotent elements and $\Gamma \subset G$ is a closed subgroup so that $G / \Gamma$ carries a $G$-invariant probability measure.

Theorem 5.1 (cf. Ratner, [12], Theorem E2). Let $\Gamma$ be an irreducible lattice in a semi-simple connected real Lie group $G, \Lambda$ and $\Lambda^{\prime}$ be lattices in some connected Lie groups $H$ and $H^{\prime}, \rho: G \rightarrow H$ and $\rho^{\prime}: G \rightarrow H^{\prime}$ be continuous homomorphisms such that the $\Gamma$-actions

$$
\gamma: h \Lambda \mapsto \rho(\gamma) h \Lambda, \quad \gamma: h^{\prime} \Lambda^{\prime} \mapsto \rho^{\prime}(\gamma) h^{\prime} \Lambda^{\prime}
$$

on $\left(H / \Lambda, m_{H / \Lambda}\right)$ and $\left(H^{\prime} / \Lambda^{\prime}, m_{H^{\prime} / \Lambda^{\prime}}\right)$ are ergodic. Assume that there exists a measurable $\Gamma$-equivariant quotient map

$$
\pi:\left(H / \Lambda, m_{H / \Lambda}\right) \longrightarrow\left(H^{\prime} / \Lambda^{\prime}, m_{H^{\prime} / \Lambda^{\prime}}\right) .
$$

Then there exists a $t \in H^{\prime}$ and a surjective continuous homomorphism $\sigma: H \rightarrow H^{\prime}$ such that

(i) $\sigma(\Lambda)$ is a finite index subgroup of $\Lambda^{\prime}$,

(ii) $\pi(h \Lambda)=t \sigma(h) \Lambda^{\prime}$ for a.e. $h \in H$,

(iii) $\sigma \circ \rho(\gamma)=t \rho^{\prime}(\gamma) t^{-1}$ for $\gamma \in \Gamma$.

If $\pi$ is one-to-one then $\sigma: H \rightarrow H^{\prime}$ is an isomorphism and $\sigma(\Lambda)=\Lambda^{\prime}$. In particular, for the above $\Gamma$-action on $\left(H / \Lambda, m_{H / \Lambda}\right)$

$$
\operatorname{Aut}^{*}\left(H / \Lambda, m_{H / \Lambda}, \Gamma\right) \cong N_{\mathrm{Aff}(H / \Lambda)}(\rho(\Gamma)) .
$$

In [15] Witte considers a more general question of a classification of all measurable equivariant quotients $\left(H / \Lambda, m_{H / \Lambda}\right) \rightarrow(Y, v)$ showing that $(Y, v)$ has an algebraic description (slightly more general than $H^{\prime} / \Lambda^{\prime}$ as above). However Theorem 5.1 suffices for our purposes. It is deduced from the more general Theorem 5.2 below by considering the measure $v$ on $H / \Lambda \times H^{\prime} / \Lambda^{\prime}$ obtained by the lift of $m_{H / \Lambda}$ to the graph of $\pi: H / \Lambda \rightarrow H^{\prime} / \Lambda^{\prime}$.

Theorem 5.2 (cf. Ratner [12], Theorem E3). Let $\Gamma \subset G, \Lambda \subset H, \Lambda^{\prime} \subset H^{\prime}$, $\rho: G \rightarrow H$ and $\rho^{\prime}: G \rightarrow H^{\prime}$ be as in Theorem 5.1. Let $v$ be a probability measure on $H / \Lambda \times H^{\prime} / \Lambda^{\prime}$ which projects onto $m_{H / \Lambda}$ and $m_{H^{\prime} / \Lambda^{\prime}}$, and is invariant and ergodic for the diagonal $\Gamma$-action

$$
\gamma:\left(h \Lambda, h^{\prime} \Lambda^{\prime}\right) \mapsto\left(\rho(\gamma) h \Lambda, \rho^{\prime}(\gamma) h^{\prime} \Lambda^{\prime}\right)
$$


Then there exist closed normal subgroups $N \triangleleft H, N^{\prime} \triangleleft H^{\prime}$, an element $t \in H^{\prime} / N^{\prime}$ and a continuous isomorphism $\sigma_{1}$ from $H_{1}:=H / N$ to $H_{1}^{\prime}:=H^{\prime} / N^{\prime}$, so that

(i) $\Lambda_{1}:=\Lambda N \subset H_{1}$ and $\Lambda_{1}^{\prime}:=\Lambda^{\prime} N^{\prime} \subset H_{1}^{\prime}$ are lattices;

(ii) there are finite index subgroups $\Delta_{1} \subseteq \Lambda_{1}, \Delta_{1}^{\prime} \subseteq \Lambda_{1}^{\prime}$ so that $\sigma_{1}\left(\Delta_{1}\right)=\Delta_{1}^{\prime}$;

(iii) $\sigma_{1} \circ \rho(\gamma)=t \rho^{\prime}(\gamma) t^{-1}$ for $\gamma \in \Gamma$;

(iv) the measure $v$ is $N \times N^{\prime}$-invariant and its projection $v_{1}$ to $H_{1} / \Lambda_{1} \times H_{1}^{\prime} / \Lambda_{1}^{\prime}$ can be obtained from the lift $m_{f}$ of $m_{H_{1} / \Delta_{1}}$ to the graph of $H_{1} / \Delta_{1} \stackrel{f}{\longrightarrow} H_{1}^{\prime} / \Delta_{1}^{\prime}$ where $f\left(h \Delta_{1}\right)=t \sigma_{1}(h) \Delta_{2}$, by $v_{1}=p_{*} m_{f}$ where $p$ is a finite-to-one projection

$$
\left(H_{1} / \Delta_{1}\right) \times\left(H_{1}^{\prime} / \Delta_{1}^{\prime}\right) \stackrel{p}{\longrightarrow}\left(H_{1} / \Lambda_{1}\right) \times\left(H_{1}^{\prime} / \Lambda_{1}^{\prime}\right) .
$$

Theorem E3 in [12] and its corollary E2 were proved by M. Ratner as an application of the main theorem ([12], Theorem 1). In all these results the acting group is assumed to be generated by Ad -unipotent elements. In order to deduce the results for actions of lattices $\Gamma \subset G$, needed for our purposes, one uses the suspension construction replacing the $\Gamma$-invariant measure $v$ on $H / \Lambda \times H^{\prime} / \Lambda^{\prime}$ by the $G$-invariant measure $\tilde{v}$ on $G \times{ }_{\Gamma} H / \Lambda \times H^{\prime} / \Lambda^{\prime}$ and applying Ratner's classification of invariant measures ([12], Theorem 1) to the action of the semi-simple group $G$ which is generated by Ad -unipotents. The reader is referred to the paper [13] of Shah (Corollary 1.4) or Witte ([15], proof of Corollary 5.8) for the precise argument.

\section{Proofs of Theorem 1.2 and Corollary 1.3}

Proof of Theorem 1.2. Let $\Gamma \subset G$ and $(X, \mu, \Gamma)$ be as in Theorem 1.2, and let $n:=$ [Out $\left.\mathrm{R}_{X, \Gamma}: \mathrm{A}^{*}(X, \Gamma)\right] \in\{1,2, \ldots, \infty\}$. If $n=1$ there is nothing to prove. If $1<n \leq \infty$ set $T_{0}=\mathrm{Id}$ and choose representatives $T_{i} \in$ Aut $_{X, \Gamma}, 1 \leq i<n$, for the cosets $\mathrm{A}^{*}(X, \Gamma) \backslash$ Out $\mathrm{R}_{X, \Gamma}$. In other words choose $T_{i}$ so that for $0 \leq i \neq j<n$ we have

$$
\left[T_{i}\right]\left[T_{j}\right]^{-1} \notin \mathrm{A}^{*}(X, \Gamma) .
$$

Since $\left[T_{i}\right] \notin \mathrm{A}^{*}(X, \Gamma)$ for $1 \leq i<n$, by Theorem 4.2 (a) there are $\tau_{i} \in$ Aut $G$ and measurable maps $\pi_{i}: X \rightarrow G / \Gamma$ satisfying

$$
\left(\pi_{i}\right)_{*} \mu=m_{G / \Gamma}, \quad \pi_{i}(\gamma \cdot x)=\gamma^{\tau_{i}} \cdot \pi_{i}(x) .
$$

It remains to prove that the map

$$
\pi: X \longrightarrow \prod_{i=1}^{n-1} G / \Gamma, \quad \pi(x)=\left(\pi_{1}(x), \pi_{2}(x), \ldots\right)
$$


takes $\mu$ onto the product measure $m_{G^{n-1} / \Gamma^{n-1}}=\prod_{i=1}^{n-1} m_{G / \Gamma}$. We shall prove by induction on finite $k$ in the range $1 \leq k<n$ that the map $\pi^{(k)}(x):=\left(\pi_{1}(x), \ldots, \pi_{k}(x)\right)$ satisfies

$$
\pi_{*}^{(k)} \mu=m_{G^{k} / \Gamma^{k}} .
$$

(Note that this is sufficient even if $n=\infty$ because the infinite product measure is determined by its values on finite cylinder sets). The case $k=1$ is covered by Theorem 4.2 (a2). Assuming (6.1) for $k-1$ we apply Theorem 5.2 to

$$
\begin{array}{rlrl}
H & :=G^{k-1} & \Lambda:=\Gamma^{k-1} & \rho:=\tau_{1} \times \cdots \times \tau_{k-1} \\
H^{\prime}:=G & \Lambda^{\prime}:=\Gamma & \rho^{\prime}:=\tau_{k}
\end{array}
$$

and the probability measure $v:=\pi_{*}^{(k)} \mu$ on $H / \Lambda \times H^{\prime} / \Lambda^{\prime}=G^{k} / \Gamma^{k}$. By the induction hypothesis $v$ projects onto $m_{H / \Lambda}$ in the first factor, and as $\left[T_{k}\right] \notin \mathrm{A}^{*}(X, \Gamma)$, $v$ projects onto $m_{H^{\prime} / \Lambda^{\prime}}$ in the second factor. If $N=H=G^{k-1}$ then necessarily $N^{\prime}=H^{\prime}=G$, so that

$$
v=m_{H / \Lambda} \times m_{H^{\prime} / \Lambda^{\prime}}=m_{G^{k} / \Gamma^{k}}
$$

proving the induction step.

It remains to show that the other alternative, namely $N \triangleleft G^{k-1}$ and $N^{\prime} \triangleleft G$ being proper normal subgroups, cannot occur. By Theorem 5.2(i) $\Lambda_{1}=\Gamma N^{\prime} \subset G / N^{\prime}$ forms a lattice in $G / N^{\prime}$ which is possible only if $N^{\prime}=\{e\}$ because $\Gamma \subset G$ is irreducible. Thus $N \triangleleft G^{k-1}$ is such that $G^{k-1} / N \cong G$ and $\Gamma^{k-1} N$ forms a lattice in $G^{k-1} / N \cong G$. This means that for some $j \in\{1, \ldots, k-1\}$

$$
\begin{aligned}
& N=\left\{\left(g_{1}, \ldots, g_{k-1}\right) \in G^{k-1} \mid g_{j}=e\right\} \\
& \sigma_{1}\left(\left(g_{1}, \ldots, g_{k-1}\right) N\right)=\sigma\left(g_{j}\right)
\end{aligned}
$$

where $\sigma \in$ Aut $G$ is such that for some $t \in G, \sigma \circ \tau_{j}(g)=t \tau_{k}(g) t^{-1}$ and $\sigma(\Delta)=\Delta^{\prime}$ for some finite index subgroups $\Delta, \Delta^{\prime} \subseteq \Gamma$. In this case the distribution $v_{1}$ of the pairs $\left(\pi_{j}(x), \pi_{k}(x)\right)$ on $G / \Gamma \times G / \Gamma$ is a projection under the finite-to-one map

$$
G / \Delta \times G / \Delta^{\prime} \longrightarrow G / \Gamma \times G / \Gamma
$$

of the measure $m_{f}$ which is a lift of $m_{G / \Delta}$ to the graph of

$$
f: G / \Delta \longrightarrow G / \Delta^{\prime}, \quad f(g \Delta)=t \sigma(g) \Delta^{\prime} .
$$

By Theorem 4.2(a3) there exist $\hat{T}_{j}$ and $\hat{T}_{k} \in$ Aut $\mathrm{R}_{X, \Gamma}$ with $\left[\hat{T}_{j}\right]=\left[T_{j}\right],\left[\hat{T}_{k}\right]=\left[T_{k}\right]$ so that for $i=j, k$ the rearrangement cocycles

$$
\alpha_{i}:=\alpha_{\hat{T}_{i}}: \Gamma \times X \longrightarrow \Gamma
$$


satisfy $\alpha_{i}(\gamma, x)=\phi_{i}(\gamma \cdot x)^{-1} \gamma^{\tau_{i}} \phi_{i}(x)$ with $\pi_{i}(x)=\phi_{i}(x) \Gamma$. The structure of the distribution $v_{1}$ of $\left(\pi_{j}(x), \pi_{k}(x)\right)$ described above implies that the distribution of $\left(\phi_{j}(x)^{\sigma}\right)^{-1} \phi_{k}(x)$ on $G$ is purely atomic. Let $S:=\hat{T}_{k} \circ \hat{T}_{j}^{-1} \in$ Aut $_{X, \Gamma}$ and let $\sigma^{\prime} \in$ Aut $G$ and $\psi=\phi_{S, \sigma^{\prime}}: X \rightarrow G$ be such that

$$
\alpha_{S}(\gamma, x)=\psi(\gamma \cdot x)^{-1} \gamma^{\sigma^{\prime}} \psi(x)
$$

Applying Proposition 2.4 (a) to $\hat{T}_{k}=S \circ \hat{T}_{j}$ we obtain that for all $\gamma \in \Gamma$ and $\mu$-a.e. $x \in X$

$$
\begin{aligned}
\phi_{k}(\gamma \cdot x)^{-1} \gamma^{\tau_{k}} \phi_{k}(x) & =\alpha_{k}(\gamma, x)=\alpha_{S}\left(\alpha_{j}(\gamma, x), \hat{T}_{j}(x)\right) \\
& =\psi\left(\alpha_{j}(\gamma, x) \cdot \hat{T}_{j}(x)\right)^{-1} \alpha_{j}(\gamma, x)^{\sigma^{\prime}} \psi\left(\hat{T}_{j}(x)\right) \\
& =\psi\left(\hat{T}_{j}(\gamma \cdot x)\right)^{-1}\left(\phi_{j}(\gamma \cdot x)^{-1} \gamma^{\tau_{j}} \phi_{j}(x)\right)^{\sigma^{\prime}} \psi\left(\hat{T}_{j}(x)\right) \\
& =\left(\phi_{j}(\gamma \cdot x)^{\sigma^{\prime}} \psi\left(\hat{T}_{j}(\gamma \cdot x)\right)\right)^{-1} \gamma^{\sigma^{\prime} \circ \tau_{j}}\left(\phi_{j}(x)^{\sigma^{\prime}} \psi\left(\hat{T}_{j}(x)\right)\right) .
\end{aligned}
$$

Replacing $\sigma^{\prime}$ by $\sigma \in$ Aut $G$ (so that $\tau_{k}=\sigma \circ \tau_{j}$ ) and changing $\psi=\phi_{S, \sigma^{\prime}}$ to $\phi_{S, \sigma}$ accordingly, we deduce that

$$
\begin{aligned}
\phi_{k}(x) & =\phi_{j}(x)^{\sigma} \phi_{S, \sigma}\left(\hat{T}_{j}(x)\right) \\
\left(\phi_{j}(x)^{\sigma}\right)^{-1} \phi_{k}(x) & =\phi_{S, \sigma}\left(\hat{T}_{k}(x)\right) .
\end{aligned}
$$

Since the distribution of $\left(\phi_{j}(x)^{\sigma}\right)^{-1} \phi_{k}(x)$ is purely atomic, it follows from Theorem 4.2 (b) that $[S] \in \mathrm{A}^{*}(X, \Gamma)$ and

$$
[S]=\left[\hat{T}_{k} \circ \hat{T}_{j}^{-1}\right]=\left[T_{k}\right]\left[T_{j}\right]^{-1} \in \mathrm{A}^{*}(X, \Gamma)
$$

contrary to the choice of $\left[T_{i}\right]$-s. Hence the induction step is verified and the proof of Theorem 1.2 is completed.

Proof of Corollary 1.3. Suppose that $\left[\right.$ Out $\left.\mathrm{R}_{X, \Gamma}: \mathrm{A}^{*}(X, \Gamma)\right] \geq n>1$. Theorem 1.2 provides a $\Gamma$-equivariant quotient map

$$
\pi:(X, \mu, \Gamma) \longrightarrow\left(G^{n-1} / \Gamma^{n-1}, m_{G^{n-1} / \Gamma^{n-1}}, \Gamma\right)
$$

where in the right hand side $\Gamma$ acts diagonally in each of the factors $\left(G / \Gamma, m_{G / \Gamma}, \Gamma^{\tau_{i}}\right)$. For diagonal actions the entropy is additive, so for every $\gamma \in \Gamma$ one has

$$
\begin{aligned}
h(X, \gamma) & \geq h\left(G^{n-1} / \Gamma^{n-1}, m_{G^{n-1} / \Gamma^{n-1}}, \gamma\right) \\
& =\sum_{i=1}^{n-1} h\left(G / \Gamma, m_{G / \Gamma}, \gamma^{\tau_{i}}\right)=(n-1) \cdot \chi(\operatorname{Ad} \gamma)
\end{aligned}
$$

which gives (1.1). 
In the context of smooth actions of $\Gamma$ on a compact $d$-manifold $X$ another application of superrigidity for cocycles allows to express the entropies $h(X, \mu, \gamma)$ of elements $\gamma \in \Gamma$ via eigenvalues of $d$-dimensional $G$-representations. More precisely, (see Furstenberg [4], Theorem 8.3, or Zimmer [17], 9.4.15) either $h(X, \mu, \gamma)=0$ for all $\gamma \in \Gamma$, or $h(X, \mu, \gamma)=\chi(\rho(\gamma)), \gamma \in \Gamma$, for some representation $\rho: G \rightarrow$ $\mathrm{GL}_{d}(\mathbb{C})$. In particular one has

$$
\inf _{\gamma} \frac{h(X, \mu, \gamma)}{\chi(\operatorname{Ad} \gamma)} \leq \max _{\operatorname{dim} \rho \leq d} \inf _{\gamma} \frac{\chi(\rho(\gamma))}{\chi(\operatorname{Ad} \gamma)} .
$$

Let us point out that in the above cited references the $\Gamma$-action on $X$ and the measure $\mu$ were assumed to be $C^{2}$-smooth, in order to apply Pesin's formula. However for the inequality (6.2) one only needs the upper bound

$$
h(X, \mu, \gamma) \leq \max _{\operatorname{dim} \rho \leq d} \chi(\rho(\gamma)), \quad \gamma \in \Gamma
$$

which, being based on Margulis-Ruelle inequality, holds under $C^{1}$-assumption on the action and does not require any regularity assumptions on the measure $\mu$.

Using Borel's density theorem one may extend the inf in (6.2) from $\gamma \in \Gamma$ to $g \in G$, obtaining the claimed estimate

$$
\text { [Out } \left.\mathrm{R}_{X, \Gamma}: \mathrm{A}^{*}(X, \Gamma)\right] \leq 1+W_{G}(d) .
$$

For a given $G$ the function $W_{G}(d)$ can be computed explicitly in terms of the weights of irreducible representations, but here let us confine the discussion to a general estimate $W_{G}(d) \leq d^{2} / 8$, suggested to me by Dave Witte, whom I would like to thank. For $k \geq 2$ let $\sigma_{k}$ denote the (unique !) irreducible representation $\sigma_{k}$ of $\mathrm{SL}_{2}(\mathbb{R})$ in dimension $k$. If $h$ denotes the element $\operatorname{diag}\left(e, e^{-1}\right) \in \mathrm{SL}_{2}(\mathbb{R})$, then the eigenvalues of $\sigma_{k}(h)$ are $\left\{e^{k+1-2 i} \mid i=1, \ldots, k\right\}$ so that

$$
\chi\left(\sigma_{k}(h)\right)=\sum_{i \leq k / 2}(k+1-2 i) \leq k^{2} / 4 .
$$

Given a $d$-dimensional $G$-representation $\rho$ choose a subgroup $\mathrm{SL}_{2}(\mathbb{R}) \simeq G_{0} \subset G$, and let $g \in G$ correspond to $h \in G_{0}$ above. The restriction $\left.\rho\right|_{G_{0}}$ of $\rho$ to $G_{0}$ splits as a direct sum of irreducible $G_{0}$-representations $\sigma_{d_{i}}$ with $\sum d_{i}=d$. Thus

$$
\chi(\rho(g))=\sum \chi\left(\sigma_{d_{i}}(h)\right) \leq 1 / 4 \sum d_{i}^{2} \leq d^{2} / 4 .
$$

At the same time $\chi\left(\operatorname{Ad}_{G}(g)\right) \geq \chi\left(\operatorname{Ad}_{S_{2}(\mathbb{R})}(h)\right)=2$, which gives

$$
W_{G}(d) \leq d^{2} / 8 .
$$




\section{Standard examples without $G / \Gamma$ quotients}

In this section we prove 1.4-1.8 applying Theorem 1.1.

Proof of Theorem 1.4. Let us first verify the ergodicity and aperiodicity of the $\Gamma$ action on $\mathbb{T}^{N}$. Let $f \in L^{2}\left(\mathbb{T}^{N}\right) \mapsto \hat{f} \in \ell^{2}\left(\mathbb{Z}^{N}\right)$ denote the Fourier transform. For $A \in \mathrm{SL}_{N}(\mathbb{Z})$ one has $\widehat{f \circ A}=A^{t} \hat{f}$. Therefore if $f \in L^{2}\left(\mathbb{T}^{N}\right)$ is an invariant vector for a subgroup $\Lambda \subset \mathrm{SL}_{N}(\mathbb{Z})$ then $\hat{f} \in \ell^{2}\left(\mathbb{Z}^{N}\right)$ is a $\Lambda^{t}$-invariant vector, and $\hat{f}$ is supported on finite $\Lambda^{t}$-orbits on $\mathbb{Z}^{N}$. Thus if $\Gamma$ fails to act ergodically on $\mathbb{T}^{N}$, then $\rho(\Gamma)^{t}$ has a non-trivial finite orbit on $\mathbb{Z}^{N}$, and for some finite index subgroup $\Gamma^{\prime} \subseteq \Gamma$ there is a non-trivial fixed vector for $\rho\left(\Gamma^{\prime}\right)^{t}$ in $\mathbb{Z}^{N} \subset \mathbb{R}^{N}$. Since $\rho: G \rightarrow \operatorname{SL}_{N}(\mathbb{R})$ is rational, Borel's density theorem implies that all of $\rho(G)^{t} \subset \mathrm{SL}_{N}(\mathbb{R})$ has a nontrivial fixed vector, and since $\rho(G)$ is totally reducible $\rho(G)$ also has non-trivial fixed vectors contrary to the assumption. Thus $\Gamma$ acts ergodically on $\mathbb{T}^{N}$, and since the arguments apply to any finite index subgroup of $\Gamma$, this action is aperiodic.

The $\Gamma$-action on $\mathbb{T}^{N}$ can be assumed to be free. Indeed $\operatorname{SL}_{N}(\mathbb{Z})$ acts freely $(\bmod 0)$ on $\mathbb{T}^{N}$ and so does $\rho(\Gamma) \cong \Gamma$.

Next we claim that the system $\left(\mathbb{T}^{N}, \Gamma\right)$ does not have $\left(\operatorname{Ad} G / \Gamma^{\prime}, \operatorname{Ad} \Gamma\right)$ as a measurable quotient. In the case of $\Gamma \subset \mathrm{SL}_{n}(\mathbb{Z})$ acting on $\mathbb{T}^{n}, n>2$, this is easily seen from the entropy comparison: for $\gamma \in \mathrm{SL}_{n}(\mathbb{Z})$ with eigenvalues $\lambda_{1}, \ldots, \lambda_{n}$ one has

$$
h\left(\mathbb{T}^{n}, \gamma\right)=\sum_{i} \log ^{+}\left|\lambda_{i}\right|, \quad h\left(\operatorname{Ad} G / \Gamma^{\prime}, \gamma\right)=\sum_{i, j} \log ^{+}\left|\lambda_{i} / \lambda_{j}\right|
$$

where $\Gamma^{\prime}$ is any lattice in $\operatorname{Ad} G=\operatorname{PSL}_{n}(\mathbb{R})$. Since $|\operatorname{det} \gamma|=1$, i.e. $\sum \log \left|\lambda_{i}\right|=0$, one has a strict inequality $h\left(\mathbb{T}^{n}, \gamma\right)<h\left(\operatorname{Ad} G / \Gamma^{\prime}, \gamma\right)$ as soon as $\gamma$ has at least one eigenvalue off the unit circle. For the general case we resort to a more complicated argument described below.

Now Theorem 1.1 (or rather its simple modification needed to handle finite center) gives

$$
\text { Out } \mathrm{R}_{\mathbb{T}^{N}, \Gamma} \cong \mathrm{A}^{*}\left(\mathbb{T}^{N}, \Gamma\right) \cong \operatorname{Aut}^{*}\left(\mathbb{T}^{N}, \Gamma\right) / \Gamma .
$$

Evidently any $\sigma \in \mathrm{GL}_{N}(\mathbb{Z})$ which normalizes $\rho(\Gamma)$ gives rise to the map $T_{\sigma}: x \mapsto$ $\sigma(x)$ of $\mathbb{T}^{N}$ which lies in $\operatorname{Aut}^{*}\left(\mathbb{T}^{N}, \Gamma\right)$.

Claim 7.1. The correspondence $\sigma \rightarrow T_{\sigma}$ is an isomorphism

$$
N_{\mathrm{GL}_{N}(\mathbb{Z})}(\rho(\Gamma)) \cong \operatorname{Aut}^{*}\left(\mathbb{T}^{N}, \Gamma\right) .
$$

The correspondence $\sigma \rightarrow T_{\sigma}$ is clearly a monomorphism of groups. To show its surjectivity consider a general $T \in \operatorname{Aut}^{\tau}\left(\mathbb{T}^{N}, \Gamma\right)$ and let $v$ denote the lift of the Lebesgue probability measure $m_{\mathbb{T}^{N}}$ on $\mathbb{T}^{N}$ to the graph of $T$. Thus $v$ is a probability measure on $\mathbb{T}^{N} \times \mathbb{T}^{N}=\left(\mathbb{R}^{N} \times \mathbb{R}^{N}\right) /\left(\mathbb{Z}^{N} \times \mathbb{Z}^{N}\right)$ which is invariant and ergodic for 
the $(\rho \times \rho \circ \tau)(\Gamma)$-action $\gamma:(x, y) \mapsto\left(\rho(\gamma)(x), \rho\left(\gamma^{\tau}\right)(y)\right)$. Witte's Corollary 5.8 in [15] (based on Ratner's theorem) allows to conclude that $v$ is a homogeneous measure for some closed subgroup

$$
M \subseteq(\rho \times \rho \circ \tau)(\Gamma) \ltimes\left(\mathbb{R}^{N} \times \mathbb{R}^{N}\right) .
$$

The connected component $M_{0}$ of the identity of $M$ can be viewed as a subgroup of $\mathbb{R}^{N} \times \mathbb{R}^{N}$. The fact that $v$ is a lift of $m_{\mathbb{T}^{N}}$ to a graph of a m.p. bijection $T: \mathbb{T}^{N} \rightarrow \mathbb{T}^{N}$, and the fact that $\mathbb{R}^{N}$ is connected while $\mathbb{Z}^{N}$ is discrete, leads to the conclusion that $M_{0} \subset \mathbb{R}^{N} \times \mathbb{R}^{N}$ projects onto $\mathbb{R}^{N}$ in both factors in a one-to-one fashion. Hence $M_{0}=\left\{(x, \sigma(x)) \mid x \in \mathbb{R}^{N}\right\}$ where $\sigma \in$ Aut $\mathbb{R}^{N}$ which preserves $\mathbb{Z}^{N}$, i.e. $\sigma \in \mathrm{GL}_{N}(\mathbb{Z})$, and $T$ has the form: $T(x)=\sigma(x)+t$ where $t \in \mathbb{T}^{N}$ is such that

$$
\sigma \circ \rho(\gamma)(x)+t=\rho\left(\gamma^{\tau}\right)(\sigma(x)+t) .
$$

The latter means that $t$ is $\rho(\Gamma)$-fixed and $\sigma \rho(\gamma) \sigma^{-1}=\rho\left(\gamma^{\tau}\right)$. An argument similar to the one for aperiodicity of the action (based on the assumption that $\rho(G)$ has no non-trivial fixed vectors), implies that $t$ has to be trivial, so that $T$ is of the form $T_{\sigma}$ where $\sigma \in N_{\mathrm{GL}_{N}(\mathbb{Z})}(\rho(\Gamma))$. The claim is proved.

It remains to show that $\mathbb{T}^{N}$ does not have $\operatorname{Ad} G / \Gamma^{\prime}$ as a measurable $\Gamma$-equivariant quotient. It follows from Witte's Corollary 5.8 ([15]) that measurable $\Gamma$-equivariant quotients of $\mathbb{T}^{N}=\mathbb{R}^{N} / \mathbb{Z}^{N}$ have the form $K \backslash \mathbb{R}^{N} / \Lambda$ where $\mathbb{Z}^{N} \subseteq \Lambda \subseteq \mathbb{R}^{N}$ is a closed $\Gamma$-invariant subgroup and $K$ is a closed subgroup of $\operatorname{Aff}\left(\mathbb{R}^{N} / \Lambda\right)$ centralizing $\Gamma$; moreover $K$ is acting non-ergodically on $\mathbb{R}^{N} / \Lambda$. The latter space can be identified with a quotient torus $\mathbb{T}^{n}, n \leq N$, on which $\Gamma$ still acts by automorphisms, so that $K$ becomes a subgroup of $\mathrm{GL}_{n}(\mathbb{Z}) \ltimes \mathbb{T}^{n}$. We claim that the $\Gamma$-action on $K \backslash \mathbb{T}^{n}$ cannot be measurably isomorphic to the $\Gamma$-action on $\operatorname{Ad} G / \Gamma^{\prime}$ because the former cannot be extended to a $G$-action. In fact the $\Gamma$-action on $K \backslash \mathbb{T}^{n}$ cannot be extended to a measurable action of the smaller group - the commensurator

$$
\Delta:=\operatorname{Comm}_{G}(\Gamma)=\left\{g \in G \mid\left[\Gamma: g^{-1} \Gamma g \cap \Gamma\right]<\infty\right\}
$$

which is a dense subgroup in $G$ (this follows from Margulis' arithmeticity results [10]). Indeed, let $g \mapsto T_{g}, g \in \Delta$, denote a hypothetical extension of the $\Gamma$-action on $K \backslash \mathbb{T}^{n}$ to some measure-preserving $\Delta$-action. For any $g \in \Delta$ there are finite index subgroups $\Gamma_{1}, \Gamma_{2} \subseteq \Gamma$ so that $\tau_{g}: \gamma \mapsto g \gamma g^{-1}$ is an isomorphism $\Gamma_{1} \rightarrow \Gamma_{2}$. Thus $T_{g}$ satisfies $T_{g}(\gamma \cdot x)=\tau_{g}(\gamma) \cdot T_{g}(x)$ for a.e. $x \in K \backslash \mathbb{T}^{n}$ and all $\gamma \in \Gamma_{1}$. Arguing as in the proof of Claim 7.1 one shows that such $T_{g}$ has to have an "algebraic" form, i.e. to be induced by a linear map $\rho(g) \in \mathrm{SL}_{n}(\mathbb{R})$ which has to preserve the lattice $\mathbb{Z}^{n}$. The fact that the embedding $\Gamma \rightarrow \mathrm{SL}_{n}(\mathbb{Z})$ cannot be extended to the commensurator $\Delta \supset \Gamma$ gives the required contradiction.

Proof of Theorem 1.5. By Margulis' Normal Subgroup Theorem ([10], (4.10)) the homomorphism $\rho: \Gamma \rightarrow K$ is actually an embedding (recall that $G$ and hence $\Gamma$ 
are assumed to be center free). Thus without loss of generality we can assume that the proper subgroup $L \subset K$ does not contain non-trivial normal factors of $K$ (otherwise dividing by these factors we still remain in the same setup). This means that the $K$-action $k_{1}: k L \rightarrow k_{1} k L$ is free $(\bmod 0)$ and so is the ergodic $\Gamma$-action $\left(K / L, m_{K / L}, \Gamma\right)$. This $\Gamma$-action is aperiodic: being connected $K$ admits no proper closed subgroups of finite index, and therefore any subgroup $\Gamma_{1} \subset \Gamma$ of finite index has a dense image $\rho\left(\Gamma_{1}\right)$ in $K$ and acts ergodically on $\left(K, m_{K}\right)$ as well as on its quotient $\left(K / L, m_{K / L}\right)$. Furthermore, such an action is irreducible - see Zimmer [16], Proposition 2.4. Clearly the discrete spectrum $\Gamma$-action on $K / L$ cannot have equivarient quotients of the form $G / \Gamma$. Hence Theorem 1.1 gives

$$
\text { Out } \mathrm{R}_{(K / L, \Gamma)}=\mathrm{A}^{*}(K / L, \Gamma) .
$$

In Theorem $1.5 \mathrm{~K} / L$ is a homogeneous space (recall that being connected $K$ has to be a Lie group). However, Theorem 1.7 (or Ratner's theorem, in general) does not apply to this situation because the acting group is not generated by Ad-unipotent elements. Yet the following general result describing $\operatorname{Aut}^{*}(K / L, \Gamma)$ can easily be obtained by direct methods.

Proposition 7.2. Let $K$ be a compact group, $\Gamma \subset K$ a dense subgroup and $L \subseteq K$ a closed subgroup. Then the left $\Gamma$-action on $\left(K / L, m_{K / L}\right)$ is ergodic and

$$
\begin{aligned}
\operatorname{Aut}(K / L, \Gamma) & \cong N_{K}(L) / L \\
\operatorname{Aut}^{*}(K / L, \Gamma) & \cong N_{\operatorname{Aff}(K / L)}(\Gamma) .
\end{aligned}
$$

Remark. In the particular case of $L=\{e\}$ the first assertion, i.e. the isomorphism $\operatorname{Aut}(K, \Gamma) \cong K$, is easy seen as follows. Any $T \in \operatorname{Aut}\left(K, m_{K}\right)$ can be written as $T(k)=k t_{k}^{-1}$ where $k \mapsto t_{k} \in K$ is a measurable map. Then $T(\gamma \cdot k)=\gamma \cdot T(k)$ translates into an a.e. identity $t_{\gamma \cdot k}=t_{k}$. Since $\Gamma$ acts ergodically on $\left(K, m_{K}\right)$ the map $k \mapsto t_{k}$ is a.e. a constant $t \in K$, i.e. $T(k)=k t$. The correspondence $T \in$ $\operatorname{Aut}(K, \Gamma) \mapsto t \in K$ is easily seen to be an isomorphism of groups.

Proof of Proposition 7.2. Given $T \in \operatorname{Aut}^{\tau}(K / L, \Gamma)$ let $v$ be the lift of $m_{K / L}$ to the graph of $T$ on $K / L \times K / L$, and let

$$
R:=\left\{\left(k_{1}, k_{2}\right) \in K \times K \mid\left(k_{1}, k_{2}\right)_{*} v=v\right\} .
$$

$R \subseteq K \times K$ forms a closed (hence compact) group, containing $\left\{\left(\gamma, \gamma^{\tau}\right) \mid \gamma \in \Gamma\right\}$. The projections $p_{i}(R)$ of $R$ to $K$ are closed and contain $\Gamma$. Hence $R$ projects onto $K$ in both coordinates. We claim that

$$
R_{1}:=\{k \in K \mid(k, e) \in R\}, \quad R_{2}:=\{k \in K \mid(e, k) \in R\}
$$


are closed normal subgroups in $K$. Indeed, for $r_{1} \in R_{1}$ and $k \in K$ there exists a $k_{2} \in K$ so that $\left(k, k_{2}\right) \in R$, and

$$
\left(k, k_{2}\right)^{-1}\left(r_{1}, e\right)\left(k, k_{2}\right)=\left(k^{-1} r_{1} k, e\right) \in R
$$

shows that $k^{-1} r_{1} k \in R_{1}$. Thus $R_{1} \triangleleft K$ and similarly $R_{2} \triangleleft K$.

Since $v$ disintegrates into Dirac measures with respect to $m_{K / L}$ under the projections $p_{i}:(K / L) \times(K / L) \rightarrow K / L$, the $R_{i}$-actions on $K / L$ should fix $m_{K / L}$-a.e. point of $K / L$. This means that $R_{i} \subseteq L$, and since $L$ is assumed not to contain non-trivial normal factors of $K, R_{i}=\{e\}$ for $i=1,2$. Hence $R$ has the form

$$
R=\{(k, \theta(k)) \mid k \in K\}
$$

for some bijection $\theta: K \rightarrow K$ which has to be a continuous isomorphism, because $R \subset K \times K$ is a closed subgroup.

By definition of $R$ for all $k \in K$ and $m_{K / L}$-a.e. $k_{1} L$, the point $\left(k k_{1} L, \theta(k) T\left(k_{1} L\right)\right)$ is on the graph of $T$, i.e. $T\left(k k_{1} L\right)=\theta(k) T\left(k_{1} L\right)$. Thus $T$ has the form $T(k L)=$ $\theta(k) t L$ where $t \in K$ is such that $\theta(L)=t L t^{-1}$. Such $T$ can also be written as $T(k L)=t \sigma(k) L$ where $\sigma(k)=t^{-1} \theta(k) t$, in which case $\sigma \in N_{\text {Aut } K}(L)$. Thus $T$ comes from an affine map $a_{\sigma, t} \in \operatorname{Aff}(K / L)$. We conclude that $\operatorname{Aut}^{*}(K / L, \Gamma)$ coincides with $N_{\mathrm{Aff}(K / L)}(\Gamma)$.

Finally, an affine map $a_{\sigma, t}$ is in $\operatorname{Aut}(K / L, \Gamma)$ if for all $\gamma \in \Gamma$ and a.e. $k L$

$$
\gamma t \sigma(k) L=t \sigma(\gamma k) L=t \sigma(\gamma) \sigma(k) L .
$$

In view of the standing assumption that $L$ does not contain normal subgroups of $K$ this means that $\sigma(\gamma)=t^{-1} \gamma t$ for $\gamma \in \Gamma$. Since $\Gamma$ is dense in $K$ we have $\sigma(k)=t^{-1} k t$ for all $k \in K$ and $\sigma(L)=L$ means $t \in N_{K}(L)$. Hence $a_{\sigma, t}: k L \mapsto\left(t t^{-1}\right) k t L=k t L$ and $t, t^{\prime} \in N_{K}(L)$ give rise to the same map of $\operatorname{Aff}(K / L)$ iff $t^{\prime} t^{-1} \in L$. This gives the desired identification

$$
\operatorname{Aut}(K / L, \Gamma) \cong N_{K}(L) / L
$$

This completes the proof of Theorem 1.5.

Proof of Theorem 1.7. By Theorem 5.1 the system $\left(H / \Lambda, m_{H / \Lambda}, \Gamma\right)$ has a $\Gamma$-equivariant quotient map

$$
\pi:\left(H / \Lambda, m_{H / \Lambda}\right) \longrightarrow\left(G / \Gamma^{\prime}, m_{G / \Gamma^{\prime}}\right)
$$

only if there exists a surjective continuous homomorphism $\sigma: H \rightarrow G$ with $\sigma(\Lambda) \subseteq$ $\Gamma^{\prime} \cong \Gamma$ and $\sigma \circ \rho(\gamma)=t \gamma t^{-1}$ for some $t \in G$. An existence of such a homomorphism $\sigma$ was explicitly excluded by the assumption, so that Theorem 1.1 gives 
Out $\left.\mathrm{R}_{H / \Lambda, \Gamma}=\mathrm{A}^{(} H / \Lambda, \Gamma\right) \cong \operatorname{Aut}^{*}(H / \Lambda, \Gamma) / \Gamma$. To identify Aut* $(H / \Lambda, \Gamma)$ we invoke Theorem 5.1 again to conclude that

$$
\operatorname{Aut}^{*}(H / \Lambda, \Gamma)=N_{\mathrm{Aff}(H / \Lambda)}(\rho(\Gamma))
$$

which presents $A^{*}(H / \Lambda, \Gamma)$ as the quotient of $N_{\mathrm{Aff}(H / \Lambda)}(\rho(\Gamma))$ by the image of $\Gamma \stackrel{\rho}{\longrightarrow} H \hookrightarrow \operatorname{Aff}(H / \Lambda)$. One also has $\operatorname{Aut}(H / \Lambda, \Gamma) \cong C_{\operatorname{Aff}(H / \Lambda)}(\rho(\Gamma))$.

Proof of Corollary 1.8. If $\rho: G \rightarrow H$ is an embedding (or isomorphism) of $G$ into another semi-simple real Lie group $H$ (center free and without compact factors) and $\Lambda \subset H$ is an irreducible lattice, then the $G$-action on $\left(H / \Lambda, m_{H / \Lambda}\right)$ is free and by Howe-Moore's theorem is not only ergodic but actually mixing. Hence also the restriction of this action to $\Gamma$-action is free and mixing, and in particular irreducible and aperiodic. The assumptions of the Corollary guarantee that there does not exits an epimorphism $\sigma: H \rightarrow G$ with $\sigma(\Lambda) \subseteq \Gamma$, so that Theorem 1.7 applies showing

$$
\text { Out } \mathrm{R}_{H / \Lambda, \Gamma}=\mathrm{A}^{*}(H / \Lambda, \Gamma) \cong \operatorname{Aut}^{*}(H / \Lambda, \Gamma) / \Gamma \cong N_{\mathrm{Aff}(H / \Lambda)}(\rho(\Gamma)) / \rho(\Gamma) .
$$

Recall that $\operatorname{Aff}(H / \Lambda)$ contains $H$ as a subgroup of finite index dividing $\mid$ Out $\Lambda \mid$. Hence, upon passing to a subgroup of index dividing $\mid$ Out $\Lambda \mid$, the group Out $\mathrm{R}_{H / \Lambda, \Gamma} \cong$ $N_{\text {Aff }(H / \Lambda)}(\rho(\Gamma)) / \rho(\Gamma)$ can be reduced to $N_{H}(\rho(\Gamma)) / \rho(\Gamma)$, which contains the centralizer $C_{H}(\rho(\Gamma))=C_{H}(\rho(G))$ as a subgroup of index dividing $\mid$ Out $\Gamma \mid$.

\section{Proof of Theorem 1.9}

Case $(\boldsymbol{G} / \boldsymbol{\Gamma}, \boldsymbol{\Gamma})$. Choose a two-sided fundamental domain $X \subset G$ for $\Gamma$ and define the transformation $I: X \rightarrow X$ by $I: x \mapsto x^{-1} \Gamma \cap X$. Note that both $X$ and $X^{-1}$ are two-sided, in particular right, fundamental domains and therefore $I$ is a measurable bijection of $X$. Moreover,

$$
I(\gamma \cdot x)=I\left(\gamma x \lambda(\gamma, x)^{-1}\right)=\lambda(\gamma, x) x^{-1} \Gamma \cap X=\lambda(\gamma, x) \cdot I(x)
$$

which means that $I \in$ Aut $\mathrm{R}_{(G / \Gamma, \Gamma)}$ and the corresponding rearrangement cocycle $\alpha_{I}$ is $\lambda=\lambda_{X}: \Gamma \times X \rightarrow \Gamma$. Observe that

$$
\gamma \cdot x=\gamma x \lambda(\gamma, x)^{-1} \text { means that } \lambda(\gamma, x)=(\gamma \cdot x)^{-1} \gamma x
$$

(with the usual multiplication in $G$ on the right hand side), so that the embedding $X \rightarrow G$ is precisely the "straightening map" $\phi$ corresponding to the cocycle $\alpha_{I}=\lambda_{X}$ and the trivial automorphism $\tau_{0}: \gamma \mapsto \gamma$; in other words $\phi_{I, \tau_{0}}(x)=x$. From Theorem 4.2 (a1) we conclude that $[I] \notin \mathrm{A}^{*}(G / \Gamma, \Gamma)$ and therefore

$$
\left[\text { Out } \mathrm{R}_{(G / \Gamma, \Gamma)}: \mathrm{A}^{*}(G / \Gamma, \Gamma)\right] \geq 2
$$


while Theorem 1.2 (or Corollary 1.3) show that this index is at most two proving an equality in (8.1). Theorem 5.1 gives

$$
\operatorname{Aut}^{*}(G / \Gamma, \Gamma) \cong N_{\mathrm{Aff}(G / \Gamma)}(\Gamma) .
$$

Note that an affine map $a_{\sigma, t} \in \operatorname{Aff}(G / \Gamma)\left(a_{\sigma, t}: g \Gamma \mapsto t \sigma(g) \Gamma\right.$ where $\sigma \in N_{\text {Aut } G}(\Gamma)$ and $t \in G$ ) satisfies

$$
a_{\sigma, t}(\gamma \cdot g \Gamma)=\gamma^{\tau} \cdot a_{\sigma, t}(g \Gamma)
$$

iff $\sigma(\gamma)=t^{-1} \tau(\gamma) t$, in particular $t \in N_{G}(\Gamma)$. Thus

$$
\operatorname{Aut}^{*}(G / \Gamma, \Gamma) \cong N_{\mathrm{Aff}(G / \Gamma)}(\Gamma) \cong N_{\mathrm{Aut} G}(\Gamma),
$$

with $g \Gamma \mapsto g^{\tau} \Gamma, \tau \in N_{\text {Aut } G}(\Gamma) \cong$ Aut $\Gamma$, giving all twisted action automorphisms. Hence $\mathrm{A}^{*}(G / \Gamma, \Gamma) \cong$ Aut $\Gamma / \Gamma \cong$ Out $\Gamma$. Since this group commutes with $[I]$, we obtain

$$
\text { Out } \mathrm{R}_{(G / \Gamma, \Gamma)} \cong \mathbb{Z} / 2 \mathbb{Z} \times \text { Out }(\Gamma)
$$

as claimed.

Before turning to the systems $\left(G^{n} / \Gamma^{n}, \Gamma\right)$ for general finite $n \geq 1$, observe that $G / \Gamma$ can be viewed as the factor of $G_{e}^{2}:=\left\{\left(g, g^{-1}\right) \in G \times G \mid g \in G\right\}$ modulo the relation $\left(g, g^{-1}\right) \sim\left(g \gamma_{1}, \gamma_{1}^{-1} g\right), \gamma_{1} \in \Gamma$. With this identification $G / \Gamma \cong\left(G_{e}^{2} / \sim\right)$ the left $\Gamma$-action on $G / \Gamma$ corresponds to the quotient of the action $\gamma:\left(g, g^{-1}\right) \mapsto$ $\left(\gamma g, g^{-1} \gamma^{-1}\right)$ modulo $\sim$, while the map $I$ arises from the flip $\left(g, g^{-1}\right) \mapsto\left(g^{-1}, g\right)$.

Case $\left(G^{n} / \Gamma^{n}, \Gamma\right), n \in \mathbb{N}$. Given a general finite $n$ consider the set

$$
G_{e}^{n+1}:=\left\{\left(g_{0}, \ldots, g_{n}\right) \in G_{e}^{n+1} \mid g_{0} \cdots g_{n}=e\right\}
$$

with the natural measure and an equivalence $\sim$ defined by

$$
\left(g_{0}, g_{1}, \ldots, g_{n-1}, g_{n}\right) \sim\left(g_{0} \gamma_{1}^{-1}, \gamma_{1} g_{1} \gamma_{2}^{-1}, \ldots, \gamma_{n-1} g_{n} \gamma_{n}^{-1}, \gamma_{n} g_{n}\right)
$$

for $\gamma_{1}, \ldots, \gamma_{n} \in \Gamma$. The map $p: G_{e}^{n+1} \rightarrow(G / \Gamma)^{n}=G^{n} / \Gamma^{n}$ given by

$$
p:\left(g_{0}, \ldots, g_{n}\right) \mapsto\left(g_{0} \Gamma, g_{0} g_{1} \Gamma, \ldots, g_{0} g_{1} \cdots g_{n-1} \Gamma\right)
$$

factors through a bijection $q:\left(G_{e}^{n+1} / \sim\right) \rightarrow G^{n} / \Gamma^{n}$. Note that the following $\Gamma$ action on $G_{e}^{n+1}$

$$
\gamma:\left(g_{0}, g_{1}, \ldots, g_{n-1}, g_{n}\right) \mapsto\left(\gamma g_{0}, g_{1}, \ldots, g_{n-1}, g_{n} \gamma^{-1}\right)
$$

descends to an action on $\left(G_{e}^{n+1} / \sim\right)$ which is isomorphic, via $q$, to the diagonal $\Gamma$-action on $G^{n} / \Gamma^{n}$

$$
\gamma:\left(g_{1} \Gamma, \ldots, g_{n} \Gamma\right) \mapsto\left(\gamma g_{1} \Gamma, \ldots, \gamma g_{n} \Gamma\right) .
$$


The cyclic permutation $\tilde{T}$ of order $(n+1)$

$$
\tilde{T}:\left(g_{0}, g_{1}, \ldots, g_{n-1}, g_{n}\right) \mapsto\left(g_{1}, g_{2}, \ldots, g_{n}, g_{0}\right)
$$

is easily seen to preserve the $\Gamma$-orbits on $\left(G_{e}^{n+1} / \sim\right) \cong G^{n} / \Gamma^{n}$ and thereby defines a relation automorphism $T \in$ Aut $\mathrm{R}_{G^{n}} / \Gamma^{n}, \Gamma$ with $\left[T^{n+1}\right] \in \mathrm{A}\left(G^{n} / \Gamma^{n}, \Gamma\right)$.

We would like to present $T$ as an explicit transformation of $\left(G^{n} / \Gamma^{n}, m_{G^{n} / \Gamma^{n}}\right)$ as follows. The cocycle $\lambda_{X}: \Gamma \times X \rightarrow \Gamma$ corresponding to the two-sided fundamental domain $X \subset G$ can be extended to a cocycle of $G$, i.e. $\lambda=\lambda_{X}: G \times X \rightarrow \Gamma$ still defined by $g x \in X \lambda(g, x)$. The left $G$-action on $X \cong G / \Gamma$ can thus be written as

$$
g \cdot x=g x \lambda(g, x)^{-1}
$$

where on the right hand side we use the usual multiplication in $G$. Using these notations and viewing $x \in X \subset G$ both as points of the space $X$ and as $G$-elements one obtains an explicit form for $T$ :

$$
T:\left(x_{1}, \ldots, x_{n}\right) \mapsto\left(x_{1}^{-1} \cdot x_{2}, x_{1}^{-1} \cdot x_{3}, \ldots, x_{1}^{-1} \cdot x_{n}, I\left(x_{1}\right)\right) .
$$

Observe that

$$
\begin{aligned}
T\left(\gamma \cdot\left(x_{1}, \ldots, x_{n}\right)\right) & =T\left(\gamma x_{1} \lambda\left(\gamma, x_{1}\right)^{-1}, \ldots, \gamma x_{n} \lambda\left(\gamma, x_{n}\right)^{-1}\right) \\
& =\left(\lambda\left(\gamma, x_{1}\right) x_{1}^{-1} \cdot x_{2}, \lambda\left(\gamma, x_{1}\right) x_{1}^{-1} \cdot x_{3}, \ldots, \lambda\left(\gamma, x_{1}\right) \cdot I\left(x_{1}\right)\right) \\
& =\lambda\left(\gamma, x_{1}\right) \cdot T\left(x_{1}, \ldots, x_{n}\right) .
\end{aligned}
$$

Hence $T \in$ Aut $\mathrm{R}_{\left(G^{n} / \Gamma^{n}, \Gamma\right)}$ with the rearrangement cocycle being

$$
\alpha_{T}\left(\gamma,\left(x_{1}, \ldots, x_{n}\right)\right)=\lambda\left(\gamma, x_{1}\right) .
$$

A similar computation shows that for $1 \leq k \leq n$ one has

$$
\alpha_{T^{k}}\left(\gamma,\left(x_{1}, \ldots, x_{n}\right)\right)=\lambda\left(\gamma, x_{k}\right)
$$

and therefore the corresponding "straightening" map is given by

$$
\phi_{T^{k}, \tau_{0}}\left(x_{1}, \ldots, x_{n}\right)=x_{k} \in G .
$$

It now follows from Theorem 4.2 (a1) that $T^{k} \notin \mathrm{A}^{*}\left(G^{n} / \Gamma^{n}, \Gamma\right)$ for $k=1, \ldots, n$. In particular

$$
\text { [Out } \left.\mathrm{R}_{\left(G^{n} / \Gamma^{n}, \Gamma\right)}: \mathrm{A}^{*}\left(G^{n} / \Gamma^{n}, \Gamma\right)\right] \geq n+1
$$

which is, in fact, an equality due to the upper bound $(n+1)$ provided by Theorem 1.2 (or Corollary 1.3).

To identify $\mathrm{A}^{*}\left(G^{n} / \Gamma^{n}, \Gamma\right)$ we invoke the second part of Theorem 5.1 with $H:=$ $G^{n}$ and $\Lambda:=\Gamma^{n}$ and note that affine maps of $G^{n} / \Gamma^{n}$ have the form

$$
\left(g_{1} \Gamma, \ldots, g_{n} \Gamma\right) \mapsto\left(t_{1} g_{p(1)}^{\tau_{1}} \Gamma, \ldots, t_{n} g_{p(n)}^{\tau_{n}} \Gamma\right)
$$


where $p \in S_{n}$ is a permutation of $\{1, \ldots, n\}, \tau_{i} \in N_{\text {Aut } G}(\Gamma) \cong$ Aut $\Gamma$ and $t_{i} \in G$. One easily checks that such a map normalizes the diagonal $\Gamma$-action iff $\tau_{1}=\cdots=$ $\tau_{n}=\tau$ and $t_{1}=\cdots=t_{n}=t$ where $t \in N_{G}(\Gamma)$. Hence Aut ${ }^{*}\left(G^{n} / \Gamma^{n}, \Gamma\right)$ consists of the maps

$$
S_{p, \tau}:\left(g_{1} \Gamma, \ldots, g_{n} \Gamma\right) \mapsto\left(g_{p(1)}^{\tau} \Gamma, \ldots, g_{p(n)}^{\tau} \Gamma\right)
$$

where $p \in S_{n}$ and $\left.\tau \in N_{\text {Aut } G}(\Gamma)\right)$. The obvious relation $S_{p, \tau} \circ S_{p^{\prime}, \tau^{\prime}}=S_{p p^{\prime}, \tau \tau^{\prime}}$ gives Aut* $\left(G^{n} / \Gamma^{n}, \Gamma\right) \cong S_{n} \times N_{\text {Aut }}(\Gamma)$ and

$$
\mathrm{A}^{*}\left(G^{n} / \Gamma^{n}, \Gamma\right) \cong S_{n} \times\left(N_{\mathrm{Aut}}(\Gamma) / \Gamma\right) \cong S_{n} \times \text { Out }(\Gamma) .
$$

Out $\mathrm{R}_{\left(G^{n} / \Gamma^{n}, \Gamma\right)}$ is generated by $[T]$ and $\mathrm{A}^{*}\left(G^{n} / \Gamma^{n}, \Gamma\right)$, and the explicit form of $T$ and $S_{p, \tau}$ allows one to check that

$$
\text { Out } \mathrm{R}_{\left(G^{n} / \Gamma^{n}, \Gamma\right)} \cong S_{n+1} \times \text { Out }(\Gamma)
$$

as claimed.

Case $\left(G^{\infty} / \Gamma^{\infty}, \Gamma\right)$. Finally, let us turn to the case of $n=\infty$, i.e. the diagonal $\Gamma$-action on $(\bar{X}, \bar{\mu}):=\left(G / \Gamma, m_{G / \Gamma}\right)^{\mathbb{Z}}$. Choose a two-sided fundamental domain $X \subset G$, so that $\bar{X}=X^{\mathbb{Z}}$, and let $\lambda=\lambda_{X}: G \times X \rightarrow \Gamma$ and $I: X \rightarrow X$ be as before. Consider the map $T: \bar{X} \rightarrow \bar{X}$ defined by

$$
T:\left(\ldots, x_{-1}, x_{0}, x_{1}, \ldots\right) \mapsto\left(\ldots, x_{1}^{-1} \cdot x_{0}, I\left(x_{1}\right), x_{1}^{-1} \cdot x_{2}, \ldots\right)
$$

so that for $k \neq 0$

$$
\begin{cases}\left(T^{k} \bar{x}\right)_{i}:=x_{k}^{-1} \cdot x_{i+k} & i \neq 1-k \\ I\left(x_{k}\right) & i=1-k\end{cases}
$$

and observe that

$$
T^{k}(\gamma \cdot \bar{x})=\lambda\left(\gamma, x_{k}\right) \cdot T^{k}(\bar{x}) .
$$

As before, for $k \neq 0$ we have $\alpha_{T^{k}}(\gamma, \bar{x})=\lambda\left(\gamma, x_{k}\right)$ and $\phi_{T^{k}, \tau_{o}}(\bar{x})=x_{k}$ so that $[T]^{k} \notin \mathrm{A}^{*}(\bar{X}, \Gamma)$.

Claim 8.1. Out $\mathrm{R}_{\bar{X}, \Gamma}$ is generated by $[T]$ and $\mathrm{A}^{*}(\bar{X}, \Gamma)$.

(Note that in previous cases similar statement followed immediately from the upper bound provided by Corollary 1.3). Choose an $S \in \operatorname{Aut}_{\bar{X}, \Gamma} \backslash \mathrm{A}^{*}(\bar{X}, \Gamma)$ and let

$$
\pi: \bar{X} \rightarrow G / \Gamma, \quad \pi_{*} \bar{\mu}=m_{G / \Gamma}, \quad \pi(\gamma \cdot \bar{x})=\gamma^{\tau} \cdot \pi(\bar{x})
$$

be the standard quotient map provided by Theorem 4.2 .

Lemma 8.2. $\pi(\bar{x})=x_{k}^{\tau}$ for some $k \in \mathbb{Z}$ and $\tau \in N_{\text {Aut } G}(\Gamma)$. 
Proof. Denote by $\bar{v}$ the probability measure on $(G / \Gamma)^{\mathbb{Z}} \times(G / \Gamma)$ obtained by the lift of $\bar{\mu}$ to the graph of $\pi$. Fix an $r \in \mathbb{N}$, let

$$
H:=\prod_{-r}^{r} G \quad \Lambda:=\prod_{-r}^{r} \Gamma
$$

and let $p: G^{\mathbb{Z}} \rightarrow H$ be the projection on $\{-r, \ldots, r\}$-coordinates. Denote by $\bar{v}^{(r)}$ the $p \times$ Id-projection of $\bar{v}$ to $H / \Lambda \times G / \Gamma$. Then one can deduce from Theorem 5.2 that either

(i) $\bar{v}^{(r)}=m_{H / \Lambda} \times m_{G / \Gamma}$, or

(ii) there exists $k \in\{-r, \ldots, r\}, \tau \in N_{\mathrm{Aut} G}(\Gamma)$ so that for any $F \in C_{c}(H / \Lambda \times G / \Gamma)$

$$
\int F d \bar{\nu}^{(r)}=\int F\left(x_{1}, \ldots, x_{k}, \ldots, x_{n}, x_{k}^{\tau}\right) d m_{G / \Gamma}\left(x_{1}\right) \cdots d m_{G / \Gamma}\left(x_{n}\right) .
$$

As $r \rightarrow \infty$ case (i) cannot persist forever, because that would imply that $\bar{v}=$ $\bar{\mu} \times m_{G / \Gamma}$ which is impossible. On the other hand as soon as (ii) occurs, the index $k$ and $\tau \in N_{\text {Aut } G}(\Gamma)$ do not change. This proves the lemma.

With the explicit form of $\pi: \bar{X} \rightarrow G / \Gamma$ provided by Lemma 8.2 we invoke Theorem 4.2 (a3) to conclude that there exists $\hat{S} \in \operatorname{Aut}_{\bar{X}, \Gamma}$ with $[S]=[\hat{S}], \tau \in$ $N_{\text {Aut } G}(\Gamma)$ and $k \neq 0 \in \mathbb{Z}$ so that

$$
\phi_{\hat{S}, \tau}(\bar{x}) \Gamma=\left(x_{k}\right)^{\tau} \Gamma .
$$

Recalling that also for $T^{k}$ we have $\phi_{T^{k}, \tau_{0}}(\bar{x})=x_{k}$ one concludes that $[S]=[\hat{S}] \in$ $\left[T^{k}\right] \mathrm{A}^{*}(\bar{X}, \Gamma)$ using the same argument as in the proof of Theorem 1.2. This completes the proof of Claim 8.1.

Any permutation $p$ of $\mathbb{Z}$ and any $\tau \in N_{\text {Aut } G}(\Gamma)$ give rise to the map $S_{p, \tau} \in$ $\operatorname{Aut}^{\tau}(\bar{X}, \Gamma)$

$$
S_{p, \tau}:\left(g_{i} \Gamma\right)_{i \in \mathbb{Z}} \mapsto\left(g_{p(i)}^{\tau} \Gamma\right)_{i \in \mathbb{Z}}
$$

On the other hand if $S \in \operatorname{Aut}^{*}(\bar{X}, \Gamma)$ let $\bar{v}$ on $\bar{X} \times \bar{X}$ be the lift of $\bar{\mu}$ to the graph of $S$ and let $\bar{v}^{r}$ be the projection of this measure to $\prod_{-r}^{r} G / \Gamma \times \prod_{-r}^{r} G / \Gamma$. Then applying the Joining Theorem 5.2 to this finite dimensional situation successively for $r \rightarrow \infty$, one concludes that such $S$ has to be of the form $S_{p, \tau}$. Hence

$$
\begin{aligned}
\operatorname{Aut}^{*}(\bar{X}, \Gamma) & \cong S_{\infty} \times N_{\text {Aut } G}(\Gamma) \\
\mathrm{A}^{*}(\bar{X}, \Gamma) & \cong S_{\infty} \times \text { Out } \Gamma .
\end{aligned}
$$

Finally, the explicit form of $[T]$ and $\left[S_{p, \tau}\right]$ allows to conclude that

$$
\text { Out } \mathrm{R}(\bar{X}, \Gamma) \cong S_{\infty+1} \times \text { Out } \Gamma
$$


where the symbols $S_{\infty}$ and $S_{\infty+1}$ can be interpreted as the inclusion of the permutation group of $\mathbb{Z}$ in the permutation group of $\mathbb{Z} \cup\{p t\}$.

\section{Proof of Theorem 1.6}

Throughout this section $\Gamma=\operatorname{PSL}_{n}(\mathbb{Z}), G=\operatorname{PSL}_{n}(\mathbb{R})$ and $n \geq 3$. Let $S_{0}=$ $\left\{p_{1}, \ldots, p_{r}\right\}$ be a given finite set of primes and consider the ergodic $\Gamma$-action on the compact profinite group $K=\prod_{p \in S_{0}} \operatorname{PSL}_{n}\left(\mathbb{Z}_{p}\right)$. We denote $H=\prod_{p \in S_{0}} \operatorname{PSL}_{n}\left(\mathbb{Q}_{p}\right)$ and $\Lambda=\operatorname{PSL}_{n}\left(\mathbb{Z}\left[S_{0}^{-1}\right]\right) \subset H$. Then $\Lambda$ is a dense countable subgroup of locally compact totally disconnected group $H$ and $\Gamma=\Lambda \cap K$.

Following Gefter [7] we first observe that Out $\mathrm{R}_{K, \Gamma}$ contains $H$. Indeed restricting the type $\mathrm{II}_{\infty}$ relation $\mathrm{R}_{H, \Lambda}$ to $K$ we obtain a type $\mathrm{II}_{1}$ relation $\mathrm{R}_{K, \Gamma}=\mathrm{R}_{H, \Lambda} \cap(K \times K)$ and

$$
\text { Out } \mathrm{R}_{K, \Gamma} \cong \text { Out } \mathrm{R}_{H, \Lambda} \supseteq \mathrm{A}(H, \Lambda) \cong H
$$

using the straight forward $\mathrm{II}_{\infty}$-type generalizations of Lemmas 2.2, 2.3(a) and the remark following 7.2 respectively.

We need to find explicit representatives $T_{h} \in$ Aut $\mathrm{R}_{K, \Gamma}$ for $h \in H$, so that $h \mapsto\left[T_{h}\right]$ is the above imbedding. Since $K$ is open and $\Lambda$ is dense in $H$, given any $h \in H$, there exist $\lambda_{0} \in \Lambda$ and $k_{0} \in K$ so that $h=\lambda_{0} k_{0}$. The maps

$$
\tilde{T}_{h}: x \mapsto x h \quad \text { and } \quad \tilde{T}_{h}^{\prime}: x \mapsto \lambda_{0}^{-1} x h \quad(x \in H)
$$

are in Aut $\mathrm{R}_{H, \Lambda}$ and $\left[\tilde{T}_{h}\right]=\left[\tilde{T}_{h}^{\prime}\right] \in$ Out $\mathrm{R}_{H, \Lambda}$. Denoting the open compact subgroup $\lambda_{0} K \lambda_{0}^{-1} \cap K$ by $K_{1}$, note that

$$
\tilde{T}_{h}^{\prime}\left(K_{1}\right) \subset K \quad \text { because } \tilde{T}_{h}^{\prime}(x)=\lambda_{0}^{-1} x \lambda_{0} k_{0} \in\left(\lambda_{0}^{-1} K_{1} \lambda_{0}\right) k_{0} \subset K .
$$

Thus for $x, y \in K_{1}$ we have

$$
(x, y) \in \mathrm{R}_{K, \Gamma}=\left.\mathrm{R}_{H, \Lambda}\right|_{K} \quad \text { iff }\left.\quad\left(\tilde{T}_{h}^{\prime}(x), \tilde{T}_{h}^{\prime}(y)\right) \in \mathrm{R}_{H, \Lambda}\right|_{K}=\mathrm{R}_{K, \Gamma} .
$$

Therefore $\left.\tilde{T}_{h}^{\prime}\right|_{K_{1}}$ is a restriction of some $T_{h} \in$ Aut $\mathrm{R}_{K, \Gamma}$, with its outer class $\left[T_{h}\right] \in$ Out $\mathrm{R}_{K, \Gamma}$ being uniquely defined by the initial $h \in H$. Denoting $\Gamma_{1}:=\lambda_{0} \Gamma \lambda_{0}^{-1} \cap \Gamma$ a finite index subgroup of $\Gamma$ which is dense in $K_{1}$, we observe that the restriction of the rearrangement cocycle $\alpha_{T_{h}}$ to $\Gamma_{1} \times K_{1}$ is

$$
\alpha_{T_{h}}\left(\gamma_{1}, x_{1}\right)=\lambda_{0}^{-1} \gamma_{1} \lambda_{0} \quad\left(\gamma_{1} \in \Gamma_{1} \text {, a.e. } x_{1} \in K_{1}\right) .
$$

The automorphism $\gamma_{1} \mapsto \lambda_{0}^{-1} \gamma_{1} \lambda_{0}$ of the lattice $\Gamma_{1} \subset G$ extends to an inner (given by $\lambda_{0} \in G$ ) automorphism of $G$, so in terms of the Standard Quotients Theorem 4.2 the class $[\tau] \in$ Out $G$ associated to such $\left[T_{h}\right] \in$ Out $_{K, \Gamma}$ is always trivial. On 
the other hand the transpose map $T_{0}:\left(k_{1}, \ldots, k_{r}\right) \mapsto\left(k_{1}^{t}, \ldots, k_{r}^{t}\right)$ which is clearly in Aut $\mathrm{R}_{K, \Gamma}$ defines the unique outer element $[\tau] \in$ Out $G$ (take $\tau(g)=\left(g^{t}\right)^{-1}$ ). One easily checks that the group generated by $\left[T_{0}\right]$ and $\left[T_{h}\right], h \in H$, in Out $\mathrm{R}_{K, \Gamma}$ is $\mathbb{Z} / 2$-extension of $H$.

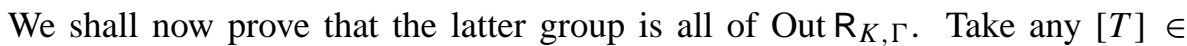
Out $\mathrm{R}_{K, \Gamma}$. Possibly composing with $T_{0}$ we may assume that $[\tau] \in$ Out $G \cong \mathbb{Z} / 2$ associated with $[T]$ is trivial, and will show that such $[T]$ is $\left[T_{h}\right]$ for some $h \in H$. Applying the Standard Quotients Theorem we may take $\tau$ to be the identity on $G$. Since $(K, \Gamma)$ cannot have $\left(G / \Gamma, m_{G / \Gamma}, \Gamma\right)$ among its measurable quotients, we deduce the following:

(1) There exists a finite $\Gamma$-orbit $F=\left\{g_{1} \Gamma, \ldots, g_{k} \Gamma\right\} \subset G / \Gamma$, and a measurable $\Gamma$-equivariant map $\pi: K \rightarrow F$ with

$$
\pi(\gamma x)=\gamma \pi(x) \quad(\gamma \in \Gamma, x \in K) .
$$

(2) Let $\Gamma_{i}=\Gamma \cap g_{i} \Gamma g_{i}^{-1}, i=1, \ldots, k$ - these are conjugate subgroups of index $k$ in $\Gamma$; the sets $X_{i}=\pi^{-1}\left(\left\{g_{i} \Gamma\right\}\right) \subset K$ are $\Gamma_{i}$-invariant and ergodic measurable subsets with $\mu\left(X_{i}\right)=1 / k$; if $K_{i}$ is the closure of $\Gamma_{i}$ in $K$ then $X_{i}=K_{i} y_{i}$ $(\bmod 0)-\operatorname{cosets}$ of $K_{i}$-s; as the latter are open and compact subsets of $K$ we obtain an open partition into disjoint sets which we still denote by $X_{i}$. Up to reordering we may assume that $X_{1}$ contains the identity of $K$, i.e. $X_{1}=K_{1}$.

(3) There exists $\hat{T} \in$ Aut $\mathrm{R}_{K, \Gamma}$ with $[\hat{T}]=[T] \in$ Out $\mathrm{R}_{K, \Gamma}$ so that

$$
\alpha_{\hat{T}}\left(\gamma_{1}, x_{1}\right)=g_{1}^{-1} \gamma_{1} g_{1} \quad\left(\gamma_{1} \in \Gamma_{1}, x_{1} \in X_{1}\right) .
$$

Note that the last formula resembles (9.1). Property (1) means that $g_{1} \in \operatorname{Comm}_{G}(\Gamma)=$ $\operatorname{PSL}_{n}(\mathbb{Q})$.

Claim 9.1. $g_{1} \in \Lambda=\operatorname{PSL}_{n}\left(\mathbb{Z}\left[1 / p_{1}, \ldots, 1 / p_{r}\right]\right)=\operatorname{PSL}_{n}\left(\mathbb{Z}\left[S_{0}^{-1}\right]\right)$.

Proof. Let us expand the notations slightly: for an arbitrary finite set $S$ of primes let

$$
K_{S}=\prod_{p \in S} \operatorname{PSL}_{n}\left(\mathbb{Z}_{p}\right), \quad \Lambda_{S}=\operatorname{PSL}_{n}\left(\mathbb{Z}\left[S^{-1}\right]\right)
$$

and let $\mu_{S}$ denote the normalized Haar measure on $K_{S}$. We shall denote by $\bar{\Gamma}_{1}^{S}$ the closure of the index $k$ subgroup $\Gamma_{1}=\Gamma \cap g_{1} \Gamma g_{1}^{-1} \subset \Gamma$ in $K_{S}$. The $\Gamma_{1}$-ergodic component $X_{1} \subset K=K_{S_{0}}$ is a coset of the open compact subgroup $\bar{\Gamma}_{1}^{S_{0}}$ of $K$ and by (2)

$$
\frac{1}{k}=\mu\left(X_{1}\right)=\mu_{S_{0}}\left(\bar{\Gamma}_{1}^{S_{0}}\right)
$$

Let $S_{1}$ be the set of primes appearing in the denominators of $g_{1} \in \operatorname{PSL}_{n}(\mathbb{Q})$, i.e. $S_{1}$ is the smallest set of primes (possibly empty) such that $g_{1} \in \Lambda_{S_{1}}$. 
It follows from the Strong Approximation Theorem that if $S=S^{\prime} \sqcup S^{\prime \prime}$ is a disjoint union of two finite sets of primes, then

$$
\overline{\Gamma_{1}} S={\overline{\Gamma_{1}}}^{S^{\prime}} \times{\overline{\Gamma_{1}}}^{S^{\prime \prime}} \subseteq K_{S^{\prime}} \times K_{S^{\prime \prime}}=K_{S},
$$

and $\overline{\Gamma_{1}} S^{\prime \prime}=K_{S^{\prime \prime}}$ if and only if $S^{\prime} \cap S_{1}=\emptyset$. On the other hand if $S_{1} \subset S^{\prime}$ then it is easy to see that

$$
\mu_{S^{\prime}}\left(\overline{\Gamma_{1}} S^{\prime}\right)=\frac{1}{\left[\Gamma: \Gamma_{1}\right]}=\frac{1}{k} .
$$

Writing $S=S_{0} \cup S_{1}=S_{0} \sqcup S_{2}$ where $S_{2}=S_{1} \backslash S_{0}$ we have

$$
\frac{1}{k}=\mu_{S_{0}}\left(\bar{\Gamma}_{1}^{S_{0}}\right) \geq \mu_{S_{0}}\left({\overline{\Gamma_{1}}}_{1}^{S_{0}}\right) \cdot \mu_{S_{2}}\left({\overline{\Gamma_{1}}}^{S_{2}}\right)=\mu_{S}\left(\bar{\Gamma}_{1}^{S}\right)=\frac{1}{\left[\Gamma: \Gamma_{1}\right]}=\frac{1}{k} .
$$

So $\mu_{S_{2}}\left(\bar{\Gamma}_{1}^{S_{2}}\right)=1$, that is $\bar{\Gamma}_{1}^{S_{2}}=K_{S_{2}}$, which means that $S_{2}=\emptyset$ and $S_{1} \subseteq S_{0}$ as claimed.

Having proved that $g_{1} \in \Lambda$, we recall that by (3) the original $T \in$ Aut $\mathrm{R}_{K, \Gamma}$ can be replaced by $\hat{T}$ with $[T]=[\hat{T}] \in$ Out $\mathrm{R}_{K, \Gamma}$ so that

$$
\hat{T}\left(\gamma_{1} x_{1}\right)=g_{1}^{-1} \gamma_{1} g_{1} \hat{T}\left(x_{1}\right)
$$

for all $\gamma_{1} \in \Gamma_{1}$ and $\mu$-a.e. $x_{1} \in X_{1}$. We have also made sure that $X_{1}=K_{1}-$ the closure of $\Gamma_{1}=\Gamma \cap g_{1} \Gamma g_{1}^{-1}$ in $K$.

Claim 9.2. $\hat{T}(k)=g_{1}^{-1} k g_{1} z_{1}$ for some fixed $z_{1} \in K$ and a.e. $k \in K_{1}$.

Proof. The map $\gamma_{1} \mapsto g_{1}^{-1} \gamma_{1} g_{1}$ is an isomorphism between finite index subgroups $\Gamma_{1} \rightarrow \Gamma_{1}^{\prime}:=g_{1}^{-1} \Gamma g_{1} \cap \Gamma$ of $\Gamma$. It extends to an isomorphism $K_{1} \rightarrow K_{1}^{\prime}$ between open compact subgroups of $K$, where $K_{1}^{\prime}$ is the closure of $\Gamma_{1}^{\prime}$ in $K$. (Note that $K_{1}=K \cap g_{1} K g_{1}^{-1}$ and $K_{1}^{\prime}=g_{1}^{-1} K g_{1} \cap K$ as subsets of $H$ ).

Let $X_{1}^{\prime}=\hat{T}\left(X_{1}\right) \subset K$. In view of (9.2), $X_{1}^{\prime}$ is one of the $\Gamma_{1}^{\prime}$-ergodic components of $X_{1}$, and therefore is a single $K_{1}^{\prime}$-coset, $X_{1}^{\prime}=K_{1}^{\prime} y$ for some $y \in X_{1}^{\prime}$. Let $R: K_{1} \rightarrow$ $K_{1}$ be the composition of the following maps

$$
K_{1}=X_{1} \rightarrow X_{1}^{\prime} \rightarrow K_{1}^{\prime} \rightarrow K_{1}, \quad R(k)=g_{1} \hat{T}(k) y^{-1} g_{1}^{-1} .
$$

In view of (9.2) we have for all $\gamma \in \Gamma_{1}$ and $\mu$-a.e. $k \in K_{1}$ :

$$
R(\gamma k)=g_{1} g_{1}^{-1} \gamma g_{1} \hat{T}(k) y^{-1} g_{1}^{-1}=\gamma R(k) .
$$

Since $\Gamma_{1}$ is dense in the compact group $K_{1}$, we have $R(k)=k k_{0}$ for some fixed $k_{0} \in K_{1}$ and a.e. $k \in K_{1}$ (see Proposition 7.2 and the following remark). This allows us to compute

$$
\hat{T}(k)=g_{1}^{-1} k k_{0} g_{1} y=g_{1}^{-1} k g_{1} z_{1} \quad \text { where } z_{1}=\left(g_{1}^{-1} k_{0} g_{1}\right) y \in K .
$$


Taking $h=g_{1} z_{1} \in H$ we observe that the map $T_{h} \in$ Aut $\mathrm{R}_{K, \Gamma}$, discussed in the first part of this section, agrees with $\hat{T}$ on a positive measure subset $K_{1} \subset K$, and therefore (as in the proof of Lemma 2.2)

$$
[T]=[\hat{T}]=\left[T_{h}\right] \in \text { Out } \mathrm{R}_{K, \Gamma}
$$

which completes the proof of the theorem.

\section{References}

[1] J. Feldman and C. C. Moore, Ergodic equivalence relations, cohomology and von Neumann algebras I, II. Trans. Amer. Math. Soc. 234 (1977), 289-324. Zbl 0369.22009 MR 0578656

[2] A. Furman, Gromov's measure equivalence and rigidity of higher rank lattices. Ann. of Math. (2) 150 (1999), 1059-1081. Zbl 0943.22013 MR 1740986

[3] A. Furman, Orbit equivalence rigidity. Ann. of Math. (2) 150 (1999), 1083-1108. Zbl 0943.22012 MR 1740985

[4] H. Furstenberg, Rigidity and cocycles for ergodic actions of semisimple Lie groups (after G. A. Margulis and R. Zimmer). Bourbaki Seminar, Vol. 1979/80, Lecture Notes in Math. 842, Springer, Berlin-New York, 1981, 273-292. Zbl 0471.22007 MR 0636529

[5] D. Gaboriau, Invariants $\ell^{2}$ de relations d'équivalence et de groupes. Inst. Hautes Études Sci. Publ. Math. 95 (2002), 93-150.. Zbl 1022.37002 MR 1953191

[6] S. L. Gefter, Ergodic equivalence relations without outer automorphisms. Dopov./ Dokl. Akad. Nauk Ukrä̈ni 11 (1993), 25-27. Zbl 0886.22004 MR 1294023

[7] S. L. Gefter, Outer automorphism group of the ergodic equivalence relation generated by translations of dense subgroup of compact group on its homogeneous space. Publ. Res. Inst. Math. Sci. 32 (1996), 517-538. Zbl 0881.28014 MR 1409801

[8] S. L. Gefter and V. Ya. Golodets, Fundamental groups for ergodic actions and actions with unit fundamental groups. Publ. Res. Inst. Math. Sci. 24 (1988), 821-847. Zbl 0684.22003 MR 1000122

[9] M. Gromov, Asymptotic invariants of infinite groups (Geometric group theory, Vol. 2). London Math. Soc. Lecture Note Ser. 182, Cambridge University Press, Cambridge, 1993. Zbl 0841.20039 MR 1253544

[10] G. A. Margulis, Discrete subgroups of semisimple Lie groups. Ergeb. Math. Grenzgeb. (3) 17, Springer-Verlag, Berlin, 1991. Zbl 0732.22008 MR 1090825

[11] N. Monod and Y. Shalom, Orbit equivalence rigidity and bounded cohomology. Ann. of Math., to appear.

[12] M. Ratner, Interactions between Ergodic theory, Lie groups and number theory. In Proceedings of the Internat. Math. Congress (Zürich 1994), Birkhäuser Verlag, Basel, 1995, 157-182. Zbl 0923.22002 MR 1403920 
[13] N. Shah, Invariant measures and orbit closures on homogeneous spaces for actions of subgroups generated by unipotent elements. In Lie groups and ergodic theory (Mumbai, 1996), Tata Inst. Fund. Res. Stud. Math. 14, Tata Inst. Fund. Res., Bombay, 1998, 229-271. Zbl 0951.22006 MR 1699367

[14] G. Stuck and R. J. Zimmer, Stabilizers for ergodic actions of higher rank semisimple groups. Ann. of Math. (2) 139 (1994), 723-747. Zbl 0836.22018 MR 1283875

[15] D. Witte, Measurable quotients of unipotent translations on homogeneous spaces. Trans. Amer. Math. Soc. 345 (1994), 577-594. Zbl 0831.28010 MR 1181187

[16] R. J. Zimmer, Ergodic actions of semisimple groups and product relations. Ann. of Math. (2) 118 (1983), 9-19. Zbl 0545.22010 MR 0707158

[17] R. J. Zimmer, Ergodic theory and semisimple groups. Monogr. in Math. 81, Birkhäuser, Boston, 1984. Zbl 0571.58015 MR 0776417

Received December 17, 2003

A. Furman, Department of Mathematics (m/c 249), University of Illinois at Chicago, 851 S. Morgan Street, Chicago, IL 60607, U.S.A.

E-mail: furman@math.uic.edu 